\title{
Deletion of Mitochondrial Uncoupling Protein 2 Exacerbates Mitochondrial Damage in Mice Subjected to Cerebral Ischemia and Reperfusion Injury under both Normo- and Hyperglycemic Conditions
}

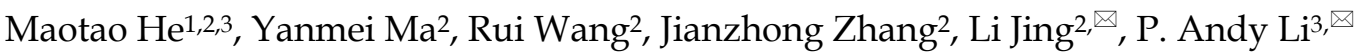 \\ 1. Department of Pathology, General Hospital of Ningxia Medical University, Yinchuan, Ningxia 750004, China \\ 2. School of Basic Medical Sciences, Department of Pathology, Ningxia Medical University; Ningxia Key Laboratory of Vascular Injury and Repair, Yinchuan, \\ Ningxia 750004, China \\ 3. Department of Pharmaceutical Sciences, Biomanufacturing Research Institute and Technological Enterprise (BRITE), College of Health and Sciences, North \\ Carolina Central University, Durham, NC 27707, USA \\ $\bowtie$ Corresponding author: Dr. P. Andy Li, Email: pli@nccu.edu; Dr. Li Jing, Email: 1203220205@qq.com \\ (c) The author(s). This is an open access article distributed under the terms of the Creative Commons Attribution License (https://creativecommons.org/licenses/by/4.0/). \\ See http://ivyspring.com/terms for full terms and conditions.
}

Received: 2020.05.14; Accepted: 2020.08.06; Published: 2020.08.25

\begin{abstract}
Deletion of mitochondrial uncoupling protein 2 (UCP2) has been shown to aggravate ischemic damage in the brain. However, the underlying mechanisms are not fully understood. The objective of this study is to explore the impact of homozygous UCP2 deletion (UCP2-/-) on mitochondrial fission and fusion dynamic balance in ischemic mice under normo- and hyperglycemic conditions. UCP2-- and wildtype mice were subjected to a 60 min middle cerebral artery occlusion (MCAO) and allowed reperfusion for $6 \mathrm{~h}, 24 \mathrm{~h}$ and $72 \mathrm{~h}$. Our results demonstrated that deletion of UCP2 enlarged infarct volumes and increased numbers of cell death in both normo- and hyperglycemic ischemic mice compared with their wildtype counterparts subjected to the same duration of ischemia and reperfusion. The detrimental effects of UCP deletion were associated with increased ROS production, elevated mitochondrial fission markers Drpl and Fis 1 and suppressed fusion markers Opal and Mfn2 in UCP2 - mice. Electron microscopic study demonstrated a marked mitochondrial swolling after $6 \mathrm{~h}$ of reperfusion in UCP2-/- mice, contrasting to a mild mitochondrial swolling in wildtype ischemic animals. It is concluded that the exacerbating effects of UCP2-- on ischemic outcome in both normo- and hyperglycemic animals are associated with increased ROS production, disturbed mitochondrial dynamic balance towards fission and early damage to mitochondrial ultrastructure.
\end{abstract}

Key words: Uncoupling protein 2; cerebral ischemia; hyperglycemia; mitochondrial dynamics; mitochondrial fission; mitochondrial ultrastructure; ROS.

\section{Introduction}

Uncoupling protein 2 (UCP2) is a member of inner mitochondrial membrane proteins, that dissipates the mitochondrial proton gradient by transporting $\mathrm{H}^{+}$across the inner membrane, thereby generating heat, stabilizing the inner mitochondrial membrane potential and reducing the formation of reactive oxygen species (ROS) [1,2]. Several studies have suggested UCP2 plays a vital role in the pathological process of neural damage after cerebral ischemia and reperfusion (I/R) $[3,4,5]$. Our previous studies have shown that deletion of the UCP2 gene exacerbates ischemic infarct volume, upregulates the protein levels of the inflammatory cytokines, and suppresses antioxidant, cell-cycle, and DNA repair genes in normoglycemic animals [6]. And that overexpression of UCP2 inhibits pro-inflammatory cytokines and activates cell cycle and cell survival factors [7].

Many studies have identified diabetes mellitus as an independent and significant risk factor for stroke as well as stroke-related mortality $[8,9,10]$. 
Both experimental and clinical studies have shown that hyperglycemia, one of the main characteristics of diabetes mellitus, further exacerbates ischemia/ reperfusion, activating cell survival pathways [11, 12], causing early damage to astrocytes and mitochondria, and inhibiting mTOR and ERK1/2 signaling [13, 14, 15]. However, the role of UCP2 in hyperglycemic ischemic damage has not been studied. In this study, we examined the effects of UCP2 deletion on ischemic outcomes in normo- and hyperglycemic mice subjected to a transient middle cerebral artery occlusion (MCAO).

Mitochondrial dynamics refers to the balance of fusion and fission in the mitochondrial network to maintain their shape, distribution, and size. Mitochondrial fission is ensured by dynamin-related protein 1 (Drp1) and fission 1 (Fis1), while Mitofusins 1, 2 (Mfn1, Mfn2) and optic atrophy 1 (Opa1) mediate mitochondrial fusion process [16]. Mitochondrial dynamics is closely associated with mitochondrial function and neurons are particularly sensitive to perturbations in mitochondrial dynamics. Accumulative evidence has revealed a close link between imbalanced mitochondrial dynamics and neurodegenerative diseases. Recent reports have suggested that mitochondrial fission is an early event required for ischemic neuronal death [17]. Mitochondrial fission occurs as early as $3 \mathrm{~h}$ after reperfusion in ischemic mice [18]. In contrast, loss of mitochondrial fusion protein Mfn2 contributes to enhanced ischemia/reperfusion injury [19]. In both in vivo and in vitro ischemic models, the expression of Mfn2 is decreased, which leads to mitochondrial dynamics imbalance and disruption of $\mathrm{Ca}^{2+}$ homeostasis [20]. Hyperglycemia further tilts the mitochondrial dynamic imbalance toward fission by increasing the levels of fission markers and decreasing fusion proteins in the early reperfusion stage [21]. However, it is unknown whether UCP2 regulates mitochondrial fission and fusion in the setting of cerebral I/R injury under normo- and hyperglycemic conditions. The objective of this study was to explore the impact of UCP2 deletion on mitochondrial dynamic balance in ischemia and reperfusion injury under both normo-and hyperglycemic conditions.

\section{Materials and Methods}

\section{Materials}

Streptozotocin (STZ) and 2,3,5-Triphenyl Tetrazolium Chloride (TTC) were obtained from Sigma. A Reactive Oxygen Species Assay Kit was purchased from Beyotime (Jiangsu, China). TUNEL Assay Kit was obtained from Roche (Mannheim, Germany). Opa1 (ab90857) and Mfn2 (ab50843) antibodies were purchased from Abcam. Fis1 (PA5-22142) antibody was purchased from Thermo Fisher scientific. Drp1 (\#8570) antibody was purchased from Cell Signaling Technology and anti- $\beta$-actin was purchased from Bios (Beijing, China).

\section{Animals and groups}

A total of 286 male UCP2-/- mice and wildtype (WT) mice were used in this study. All animal procedures were performed following the NIH Guide for Care and Use of Laboratory Animals and were approved by the Institutional Animal Care and Use Committee at Ningxia Medical University. Breeding pairs of UCP2-/-mice were obtained from the Jackson laboratory and their off-springs were genotyped. The mice were maintained in a specific pathogen-free colony of the Laboratory Animal Center of Ningxia Medical University (Yinchuan, China) with controlled temperature, humidity, and 12:12 hour light and dark cycle.

$\mathrm{UCP} 2 \%$ mice and WT mice were randomly divided into normo- and hyperglycemic groups, each consisting of four subgroups: a sham-operated control and $1 \mathrm{~h}$ MCAO plus $6 \mathrm{~h}, 24 \mathrm{~h}$, and $72 \mathrm{~h}$ of reperfusion. The animal groups and numbers used in each group are summarized in Table 1. Hyperglycemia was induced by intraperitoneal injection of STZ (120 $\mathrm{mg} / \mathrm{kg}$ ) that was freshly dissolved in $0.1 \mathrm{M}$ citrate buffered saline ( $\mathrm{pH} 4.5)$. Age-matched mice receiving the same volume of $0.1 \mathrm{M}$ citrate-buffered saline served as normoglycemic controls. The blood glucose level was measured after $72 \mathrm{~h}$ of STZ injection using Blood Glucose Meter (Boshilong, Taiwan). Animals with a blood glucose level higher than $16.8 \mathrm{mmol} / \mathrm{L}$ were designated as the hyperglycemia mice based on our previous experience [22].

\section{Ischemia and reperfusion model}

The animals were anesthetized with $3 \%$ isoflurane for induction and maintained at $1.0-1.5 \%$ during the surgical procedures. The anesthesia was delivered in a $70 \%$ nitrous oxide and $30 \%$ oxygen mixture using a facemask (MATRX VIP 3000). Cerebral ischemia was induced by middle cerebral artery occlusion (MCAO). Briefly, the internal carotid artery (ICA), external carotid artery (ECA), and the common carotid artery (CCA) were isolated through a midline incision. The right CCA was ligated, and the right ICA was temporarily closed by a loose suture. A small incision was made on the CCA and a filament (Doccol corporation, USA), which had a distal cylinder of silicon rubber with a diameter of $0.21 \pm 0.02$ $\mathrm{mm}$, was inserted into the ICA until a faint resistance was felt. After $60 \mathrm{~min}$ occlusion, the filament was withdrawn to achieve recirculation. The sham animals 
were subjected to the same surgical procedure as the MCAO mice but without occlusion of the MCA. During the surgery period, the body temperature of the mice was maintained with a heating pad and lamp and monitored by a rectal thermometer (Omron, Dalian, China). The mice were subjected to a neurological examination immediately after the animals recovered from anesthesia to judge the successful induction of MCAO model and again after $24 \mathrm{~h}$ reperfusion to compare the functional recovery between the experiential groups. The neurological deficit was scored by Zea-Longa scale: 0, no neurological deficits; 1 , failure to fully extend left paw; 2, circling to the left; 3, falling to the left; 4, unable to walk spontaneously and exhibiting depressed levels of consciousness. The animal with scores of 2 and above was selected as the successful MCAO model. Five animals were excluded due to lack of neurological deficit after MCAO. All animals were coded with a number and the people who further process the measurements and analysis were blinded to the experimental conditions.

Table 1. Summary of group and subgroups

\begin{tabular}{|c|c|c|c|c|c|}
\hline Groups & Processing & TTC & Histo & WB & TEM \\
\hline \multicolumn{6}{|l|}{ WT } \\
\hline \multicolumn{6}{|l|}{ NG } \\
\hline Sham & Sham-operated & 4 & 10 & 4 & 4 \\
\hline $6 \mathrm{~h} \mathrm{I} / \mathrm{R}$ & $\mathrm{MCAO}+$ reperfusion $6 \mathrm{~h}$ & 0 & 6 & 6 & 4 \\
\hline $24 \mathrm{~h} \mathrm{I} / \mathrm{R}$ & $\mathrm{MCAO}+$ reperfusion $24 \mathrm{~h}$ & 4 & 10 & 6 & 0 \\
\hline $72 \mathrm{~h} \mathrm{I} / \mathrm{R}$ & $\mathrm{MCAO}+$ reperfusion $72 \mathrm{~h}$ & 0 & 6 & 6 & 0 \\
\hline \multicolumn{6}{|l|}{ HG } \\
\hline Sham & Sham-operated & 0 & 10 & 4 & 4 \\
\hline $6 \mathrm{~h} \mathrm{I} / \mathrm{R}$ & $\mathrm{MCAO}+$ reperfusion $6 \mathrm{~h}$ & 0 & 6 & 6 & 4 \\
\hline $24 \mathrm{~h} \mathrm{I} / \mathrm{R}$ & $\mathrm{MCAO}+$ reperfusion $24 \mathrm{~h}$ & 4 & 10 & 6 & 0 \\
\hline $72 \mathrm{~h} \mathrm{I} / \mathrm{R}$ & $\mathrm{MCAO}+$ reperfusion $72 \mathrm{~h}$ & 0 & 6 & 6 & 0 \\
\hline \multicolumn{6}{|l|}{ UCP2 $\%$} \\
\hline \multicolumn{6}{|l|}{ NG } \\
\hline Sham & Sham-operated & 5 & 10 & 4 & 4 \\
\hline $6 \mathrm{~h} \mathrm{I} / \mathrm{R}$ & $\mathrm{MCAO}+$ reperfusion $6 \mathrm{~h}$ & 0 & 6 & 6 & 4 \\
\hline $24 \mathrm{~h} \mathrm{I} / \mathrm{R}$ & $\mathrm{MCAO}+$ reperfusion $24 \mathrm{~h}$ & 5 & 10 & 6 & 0 \\
\hline $72 \mathrm{~h} \mathrm{I} / \mathrm{R}$ & $\mathrm{MCAO}+$ reperfusion $72 \mathrm{~h}$ & 0 & 6 & 6 & 0 \\
\hline \multicolumn{6}{|l|}{ HG } \\
\hline Sham & Sham-operated & 0 & 10 & 4 & 4 \\
\hline $6 \mathrm{~h} \mathrm{I} / \mathrm{R}$ & $\mathrm{MCAO}+$ reperfusion $6 \mathrm{~h}$ & 0 & 6 & 6 & 4 \\
\hline $24 \mathrm{~h} \mathrm{I} / \mathrm{R}$ & $\mathrm{MCAO}+$ reperfusion $24 \mathrm{~h}$ & 5 & 10 & 6 & 0 \\
\hline $72 \mathrm{~h} \mathrm{I} / \mathrm{R}$ & $\mathrm{MCAO}+$ reperfusion $72 \mathrm{~h}$ & 0 & 6 & 6 & 0 \\
\hline \multicolumn{6}{|c|}{$\begin{array}{l}\text { WT: Wildtype mice; UCP2-- : knock out UCP2 mice; NG: normoglycemia; HG: } \\
\text { hyperglycemia; } 6 \mathrm{~h} \mathrm{I} / \mathrm{R}, 24 \mathrm{~h} \text { I/R and } 72 \mathrm{~h} \text { I/ R: MCAO and reperfusion } 6 \mathrm{~h}, 24 \mathrm{~h} \text {, and } \\
\text { 72h. MCAO: middle cerebral artery occlusion; TTC: } 2,3,5 \text {-triphenyltetrazolium } \\
\text { chloride; Histo: histology; WB: Western blot; TEM: transmission electron } \\
\text { microscopy. }\end{array}$} \\
\hline
\end{tabular}

\section{Anatomy of the MCA and Circle of Willis}

The animals were deeply anesthetized and transcardially perfused with $0.9 \%$ saline to flush out the blood. Mouse was injected with Indian ink (2\%) made in $20 \%$ gelatin and saline. After perfusion brain was cooled to allow gelatin solidification and fixed with $4 \%$ paraformaldehyde. Brain images were captured using a Nikon digital camera.

\section{Measurement of infarct volume and edema}

The mice were sacrificed at $24 \mathrm{~h}$ after MCAO and whole brains were dissected coronally into 1-mm brain slices using a stainless brain matrix (68707, RWD, Shenzhen). The brain slices were immediately placed into a 24-well plate and incubated with 2, 3, 5-triphenyltetrazolium chloride (TTC, $2 \%$ ) at $37^{\circ} \mathrm{C}$ for $15 \mathrm{~min}$ and then fixed in $4 \%$ paraformaldehyde. TTC stains viable brain tissue as deep red but infarcted tissues as pale color. Areas of infarcted tissue were measured using NIH Image J software (rsb.info.nih .gov/nih-image) and infarct volumes were calculated from all sections with corrections of intersection distance. The infarcted volume was expressed as the percentage of infarcted tissue relative to total brain tissue. Edema volume was semi-quantitatively analyzed after $24 \mathrm{~h}$ reperfusion. The relative edema volume (\%) was calculated as: (ipsilateral hemisphere volume - contralateral hemisphere volume) / (contralateral hemisphere volume) $\times 100 \%$.

\section{ROS detection}

Frozen mice brain sections were cultured and incubated in the dark with $10 \mu \mathrm{M} / \mathrm{L}$ of Dihydroethidium (DHE) for $30 \mathrm{~min}$ at $37^{\circ} \mathrm{C}$. Intracellular ROS production was assessed with an Olympus FluoView1000 Laser Scanning Confocal Microscope (using ex/em $\lambda=480 \mathrm{~nm} / 535 \mathrm{~nm}$ for DHE).

\section{TUNEL staining}

Terminal deoxynucleotidyl transferase mediated dUTP nick-end labeling (TUNEL) staining was used to detect apoptosis cells (Roche, \#11684795910) according to the manufacturer's protocol. The number of TUNEL-positive cells was counted in five microscopic fields at $400 \mathrm{X}$.

\section{Immunohistochemistry assay}

Paraformaldehyde-fixed and paraffin-embedded brain tissues were sectioned ( $4 \mu \mathrm{m}$ thickness). The sections were submerged in citrate buffer $(\mathrm{pH}=6.0)$ and heated at boiling temperature under a high-pressure situation for $5 \mathrm{~min}$ for antigen retrieval. The expression levels of Fis1 (1:400, PA5-22142, Thermo Fisher), Opa1(1:200, ab90857, Abcam), Drp1(1:200, \#8570, CST) and Mfn2 (1:200, ab50843, Abcam) proteins were examined in each group after primary antibody incubation overnight and secondary antibody incubation at $37^{\circ} \mathrm{C}$ for $45 \mathrm{~min}$. The reaction was visualized with DAB (ZSGB-BIO, Beijing, China) staining and then hematoxylin counterstaining the nuclei. The number of positively stained cells was counted in five microscopic fields at $400 \mathrm{X}$. 


\section{Western blot}

The brain tissues were homogenized on ice with lysis buffer. We determined the protein concentration of each protein sample to ensure that the sample volumes were consistent. Equal amounts $(50 \mu \mathrm{g})$ of protein extracts were subjected to $10 \%-12 \%$ sodium dodecyl sulfate-polyacrylamide gels electrophoresis (SDS-PAGE) and transferred to polyvinylidene fluoride membranes (Millipore). The membranes were incubated overnight at $4^{\circ} \mathrm{C}$ with the following primary antibodies: anti-Fis1 (1:1000), anti-Opa1 (1:1000), anti-Mfn2 (1:1000) and anti-Drp1 (1:1000). Then, the membranes were incubated with secondary antibodies for $1 \mathrm{~h}$ at room temperature. Imaging was performed using the BIO-RAD Imaging System with chemiluminescence detection reagents. Semi-quantitative results were obtained by measuring the optical density of the target bands and were expressed as the ratio of each targeted protein to $\beta$-actin. We make the band densities in WT+NG sham groups as 100 to compare differences in other group. Band relative densities were analyzed by NIH Image J software (rsb.info.nih.gov/nih-image).

\section{Electron Microscopic Studies}

Brains were perfusion fixed with $2 \%$ glutaraldehyde at $6 \mathrm{~h}$ of reperfusion collected from both normoglycemic and hyperglycemic animals. The brain sections were taken between Bregma 1 to $-1 \mathrm{~mm}$ and post-fixed with $4 \%$ glutaraldehyde in $0.1 \mathrm{~mol} / \mathrm{L}$ cacodylate buffer ( $\mathrm{pH}$ 7.4). The sections were then soaked in $1 \%$ osmium tetroxide in $0.1 \mathrm{M}$ cacodylate buffers for $2 \mathrm{~h}$ and stained with 1\% aqueous uranyl acetate overnight. Tissue sections were dehydrated in ascending series of ethanol to $100 \%$ followed by dry acetone and embedded in epoxy resin. Ultrathin sections were cut and counterstained with lead citrate before examination by transmission electron microscope (H7650).

\section{Statistics}

All data are presented as means \pm SD. Statistical analysis was performed using one-way ANOVA with SPSS 19.00. Student's t-test was used to analyze the difference in infarct volume between the two animal species. Statistical significance was determined as $p<$ 0.05 . The numbers of animals in each subgroup are given in Table 1 and in Figure legends.

\section{Results}

\section{Cerebral vasculature and blood glucose levels in transgenic and WT mice}

The experimental protocol is illustrated in Fig. 1A. To evaluate whether UCP2 deletion causes phenotypic changes in the cerebral vasculature, we transcardially injected Indian black ink and imaged the cerebral blood vessels (Fig. 1B). The result showed intact and correct alignment of the circle of Willis, anterior, middle, and posterior cerebral arteries with no remarkable difference between WT and UCP2-/mice. Physiological variables were measured and maintained constant. Body temperature was kept at $36.5^{\circ} \mathrm{C}$ to $37.5^{\circ} \mathrm{C}$. As expected, the blood glucose (Fig. 1C) was significantly higher in the STZ induced hyperglycemia mice than those in control group $(p<0.01)$. The average blood glucose level in the WT normal glucose group (WT+NG) was $7.70 \pm 1.32 \mathrm{mM}$, while that in the WT hyperglycemic group (WT+HG) was $22.19 \pm 6.10 \mathrm{mM}(p<0.01$ vs. WT+NG). Similarly, blood glucose concentration was $7.15 \pm 2.00 \mathrm{mM}$ in $\mathrm{UCP} 2 \%$ normal glucose group (UCP2 $/-+\mathrm{NG}$ ) and $21.91 \pm 6.31 \mathrm{mM}$ in $\mathrm{UCP} 2 \%$ hyperglycemic group $(\mathrm{UCP} 2--+\mathrm{HG})(p<0.01$ vs. $\mathrm{UCP} 2 \%+\mathrm{NG})$. However, there was no difference in blood glucose level between WT and UCP2-/- animals. Animal body weight and body temperature were the same between UCP2 $\%$ and WT under same glycemic conditions (Fig. $1 \mathrm{D}$ and $1 \mathrm{E})$.

\section{UCP2 deletion aggravated ischemic brain damage in both normo- and hyperglycemic mice.}

To determine whether the deletion of UCP2 can aggravate ischemic brain damage under both normoand hyperglycemic conditions, we examined infarct volumes and neurological deficits. First, the results showed that UCP2 deletion increased infarct volume compared to WT mice under normoglycemic condition. Focal ischemia of $60 \mathrm{~min}$ duration induced brain infarct in the striatum, without much involvement of the overlaying cortex in WT mice at $24 \mathrm{~h}$ of reperfusion. The infarct volume was significantly enlarged in UCP2 $/$ - mice compared with the WT mice, which covered both the striatum and the cortex. Second, hyperglycemia enlarged infarct volume compared with that in normoglycemic WT mice (Fig. 2A and 2B). Third, UCP2 deletion with hyperglycemia further expanded the infarct volume compared with WT mice under hyperglycemic condition. Similarly, UCP2 $\%$ mice showed a significant increase in neurological deficit scores compared with WT ischemic mice (Fig. 2C), indicating neurological deficit scores were positively correlated to the infarct size. Compared with the WT+HG group, UCP2 $\%$ + HG group had more severe neurological deficit scores after ischemia/reperfusion $(p<0.05)$. Measurement of brain edema also suggested that $\mathrm{UCP} 2 \%$ significantly increased brain edema compared to WT mice under both normo- and 
hyperglycemic ischemic conditions ( $p<0.05$, Fig. 2D). Our data demonstrate that UCP2 deletion aggravated ischemic brain damage in both normo- and hyperglycemic mice.

\section{UCP2 deletion aggravated histopathological changes and apoptotic death after ischemia under both normo- and hyperglycemic conditions.}

The pathological outcomes in the cortex are given in Figure 3A and 3B. As shown in Figure 3A, a few scattered dead neurons were observed in the sham-operated animals. Transient cerebral ischemia resulted in a mildly increased number of dead neurons in the cortex after $24 \mathrm{~h}$ of reperfusion in WT mice $(p<0.01)$. Deletion of UCP2 further increased the percentage of dead neurons in the cortex $24 \mathrm{~h}$ after reperfusion compared with the WT counterpart under both euglycemic and hyperglycemic conditions (Fig. 3A and 3B). As expected, hyperglycemia caused more ischemic cell death than normoglycemic animals in both WT and UCP2 $\%$ mice. Interestingly, Nissl staining showed a significant decrease after ischemia in WT animals with euglycemic condition. Deletion of UCP2 did not seem to further reduce to the Nissl staining in euglycemic mice. However, deletion of

A

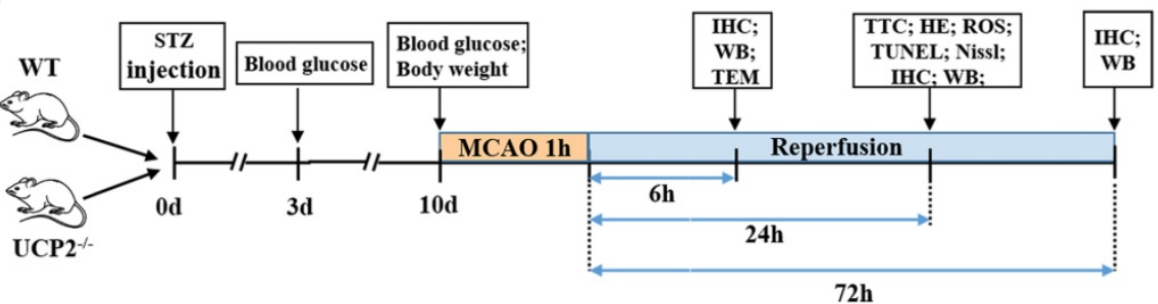

B
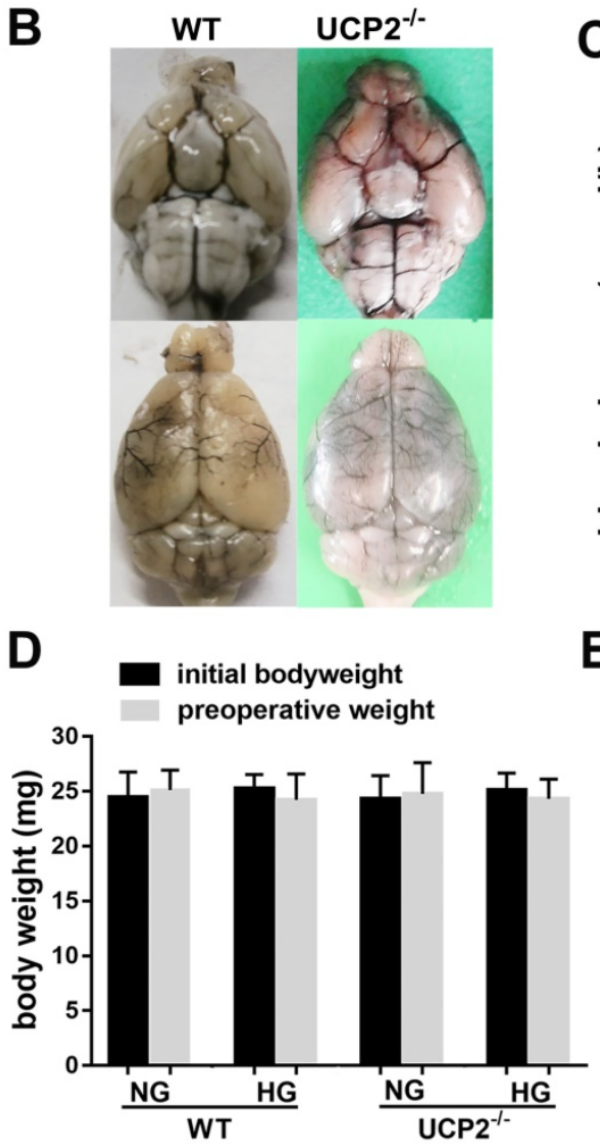

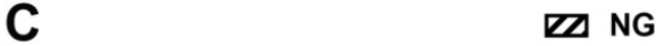

ED HG
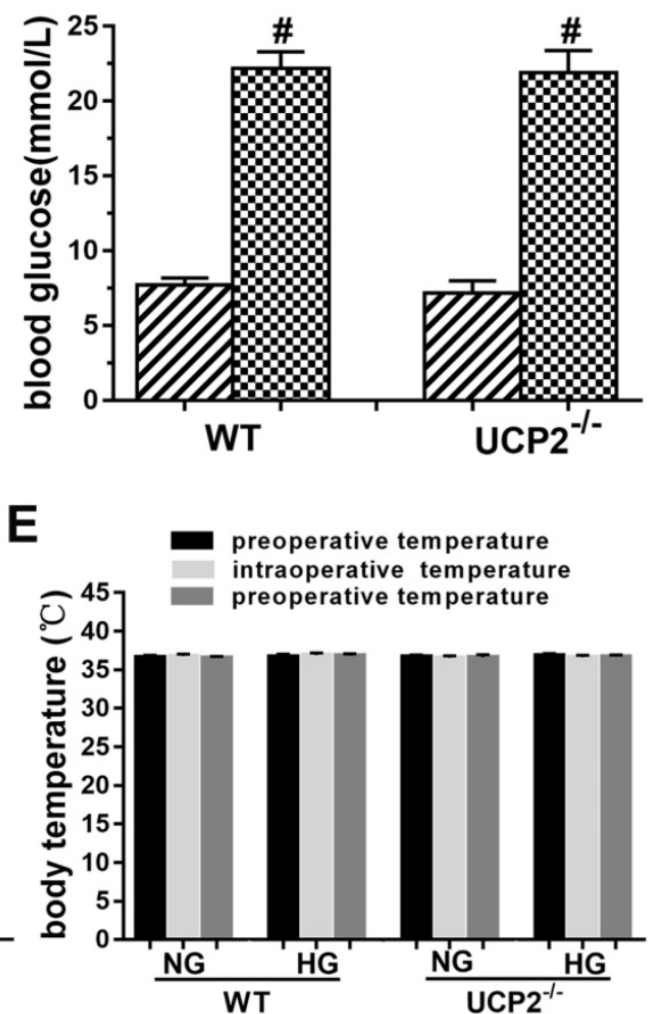

Figure 1. Effect of UCP2 deletion on the onset of high glucose in different mice. (A) This diagram illustrated the experimental design. (B) Cerebral vasculature. Mice $\left(n=3\right.$, each group) were perfused with Indian black ink to determine whether there were vascular abnormalities in the UCP2 ${ }^{-/-}$mice. The Circle of Willis, anterior cerebral arteries, middle cerebral arteries, and posterior arteries all appear normal as compared with those in WT controls. (C) Blood glucose level. The blood glucose was obviously higher in the STZ induced diabetic mice than those in control group $(p<0.01)$. There was no significant in WT mice and UCP2-- mice ( $\mathrm{n}=54$, each group). (D) Body weight. There was no significant different between initial and preoperative weight in different groups $(n=54$, each group). (E) Body temperature. There was no significant among preoperative temperature, intraoperative temperature and postoperative ( $n=54$, each group). 
UCP2 in hyperglycemic animals significantly decreased Nissl body's density compared with WT mice in hyperglycemic group (Fig. 3C and 3D). TUNEL staining images were taken from the ischemic penumbra area in the ipsilateral cortex. We selected the penumbral area because most apoptotic cells present in this area. Our results revealed ischemia increased the number of TUNEL positive cells. Similarly, hyperglycemic ischemia increased the number of TUNEL positive cells compared with normoglycemic ischemia and UCP2 deletion further elevated the numbers of TUNEL positive cells after ischemia in both normo-and hyperglycemic animals compared with the WT counterparts (Fig. 3E and 3F). The right corner of the WT+HG image was taken close to the ischemic core. There was less TUNEL positive neurons in this area probably due to the fact that severe insult in HG ischemia led to more necrotic, instead of apoptotic, cell death.

\section{UCP2 deletion enhanced ROS production in both normo- and hyperglycemic mice after ischemia.}

As shown in Figure 4A and 4B, MCAO induction caused a significant enhancement of superoxide production as detected by DHE. Consistently, hyperglycemia further increased the ROS compared with normoglycemic mice after ischemia and reperfusion at $24 \mathrm{~h}$ in the cortex and deletion of UCP2 led to a much more pronounced elevation of ROS in both normo- and hyperglycemic animals after ischemia and reperfusion injury compared with their matched WT counterparts.

\section{UCP2 deletion further exacerbated the ischemia-induced mitochondrial dynamic imbalance}

Mitochondrial dynamics constantly change between fission and fusion status. To understand the mechanism by which UCP2 modulates mitochondrial fission/fusion balance, we detected mitochondrial fission and fusion-associated proteins in cortical samples collected from animals subjected to $60 \mathrm{~min}$ of ischemia and followed by $6 \mathrm{~h}, 24 \mathrm{~h}$ and $72 \mathrm{~h}$ of reperfusion by immunohistochemistry and Western blot (Fig. 5 and Fig. 6).

The results of immunohistochemistry for Fis1 and Drp1 are given in Figure 5A-5D. The positive cells were labeled in brownish yellow color, and hematoxylin counterstaining stained the nuclei in
A
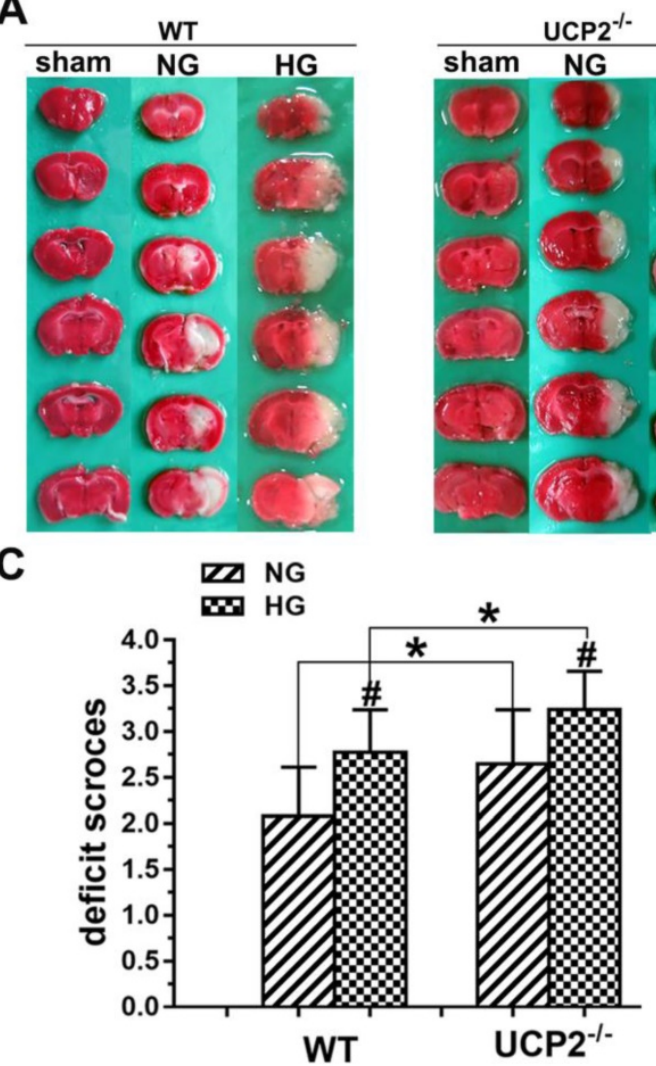

B
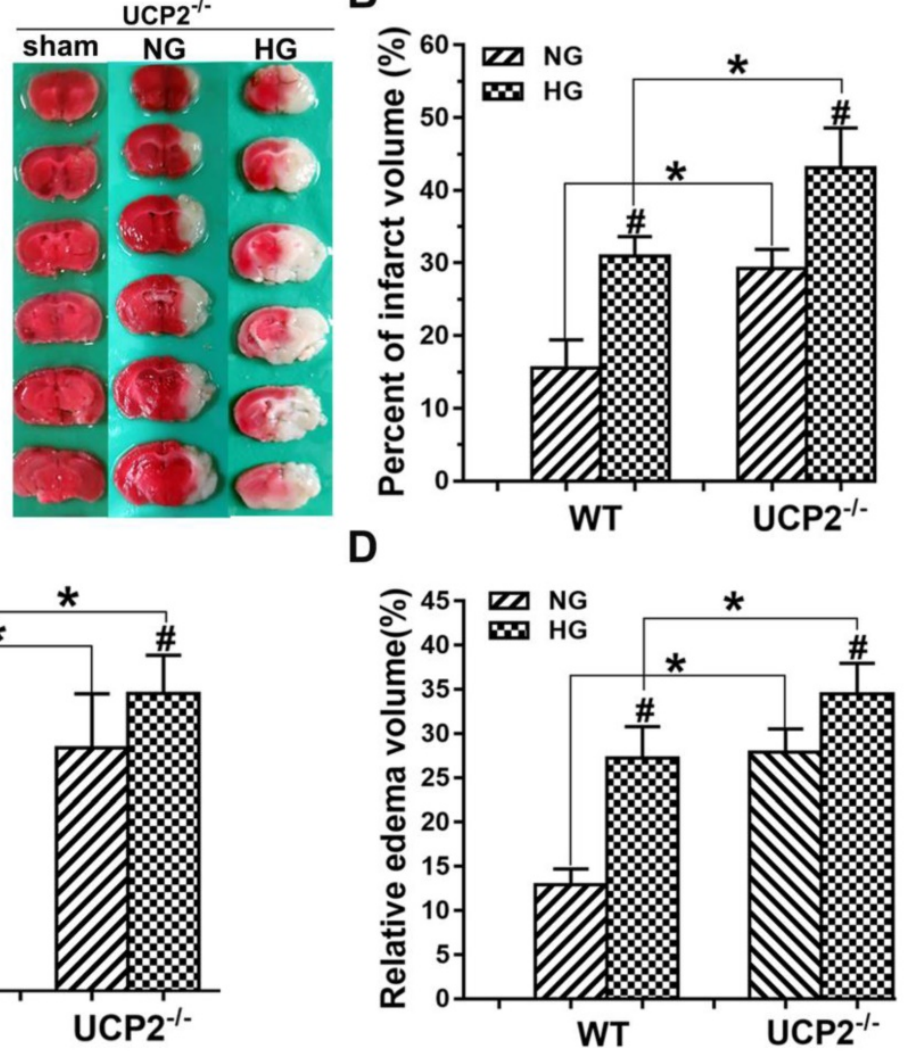

Figure 2. UCP2 deletion aggravated ischemic brain damage in hyperglycemic mice. (A) Representative TTC stained brain sections showing infarct volume (white color) at 24-h of reperfusion following $60 \mathrm{~min}$ of MCAO in WT and UCP2-/- mice. There were 4 animals for WT each group and 5 animals for UCP2-/ each group. (B) Bar graph summarizes the mean values of cerebral infarction in WT and UCP2 - mice. Infarct volume enlarged significantly in UCP2 - mice with high glucose $(\mathrm{n}=4$ in each WT group; $\mathrm{n}=$ 4 in each UCP2--- group). ${ }^{*} p<0.05$ vs. NG; ${ }^{*} p<0.05$ vs. WT. (C) Assessments of neurological deficits ( $n=20$ in each group). $\# p<0.05$ vs. NG; ${ }^{*} p<0.05$ vs. WT. (D) Quantitative analysis of edema volume. There were 4 animals for WT each group and 5 animals for UCP2-/- each group. ${ }^{\#} p<0.05$ vs. NG; ${ }^{*}<<0.05$ vs. WT, respectively. 
blue. The expression of mitochondrial fission-related proteins was evaluated by the ratio of the number of positive cells to the total number of cells. Ischemia in normoglycemic WT mice induced a significant elevation of Fis1 and its level already peaked at $6 \mathrm{~h}$ of reperfusion and maintained at that high level up to 72h (Fig. 5A and 5C). UCP2 deletion caused a further elevation of Fis1 in both normo- and hyperglycemic mice after ischemia compared with the WT mice. Changes of Drp1 were similar to those of Fis1. Thus, UCP2 deletion further pushed the Drp1 to higher levels at $24 \mathrm{~h}$ and $72 \mathrm{~h}$ in normoglycemic and $72 \mathrm{~h}$ in hyperglycemic animals than in the WT counter groups (Fig. 5B and 5D). These results were further supported by Western blot. As shown in Figure 5E-5G, I/R injury upregulated the expression of proteins related to mitochondrial fission such as Fis1 and Drp1 over reperfusion time. The trend of Fis1 changes was similar to the results of immunohistochemistry (Fig. 5E and 5F). However, the level of Drp1 reached a peak at $24 \mathrm{~h}$ in UCP2 $\%$ mice and was significantly higher than that in WT mice in hyperglycemic group (Fig. 5E and 5G).

Immunohistochemistry detection of mitochondrial fusion proteins Opa1 and Mfn2 revealed decreases of these two proteins in UCP2-mice (Fig. 6A-6D). As shown, Opa1 level decreased slightly in WT mice after $72 \mathrm{~h}$ of reperfusion, while UCP2 deletion decreased Opa1 content at $6 \mathrm{~h}$ and $24 \mathrm{~h}$ compared with WT ischemic mice (Fig. 6A and 6C). $\mathrm{UCP} 2$ deletion in hyperglycemic animals lowered Opa1 values in control and at $6 \mathrm{~h}$ of reperfusion. UCP2 deletion resulted in a significant reduction of Mfn2 at $72 \mathrm{~h}$ of reperfusion in normoglycemic ischemic
A

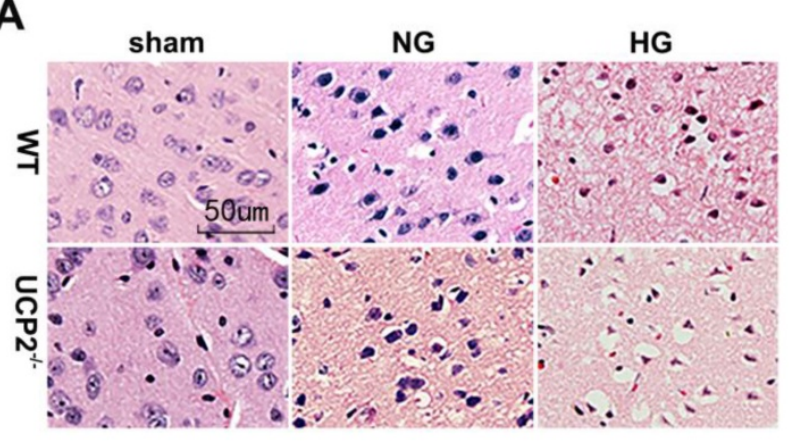

C

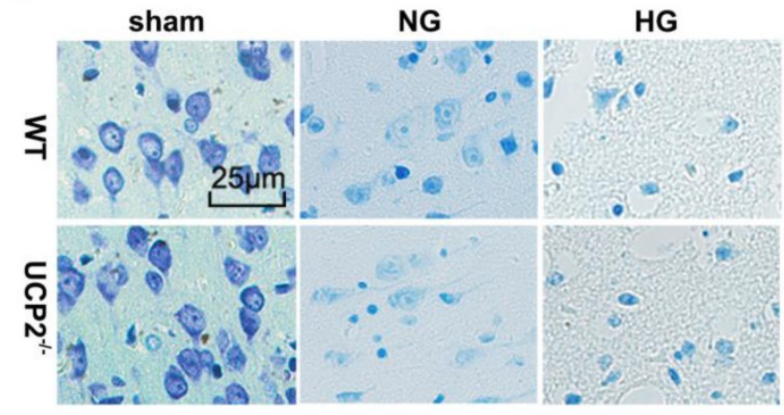

E
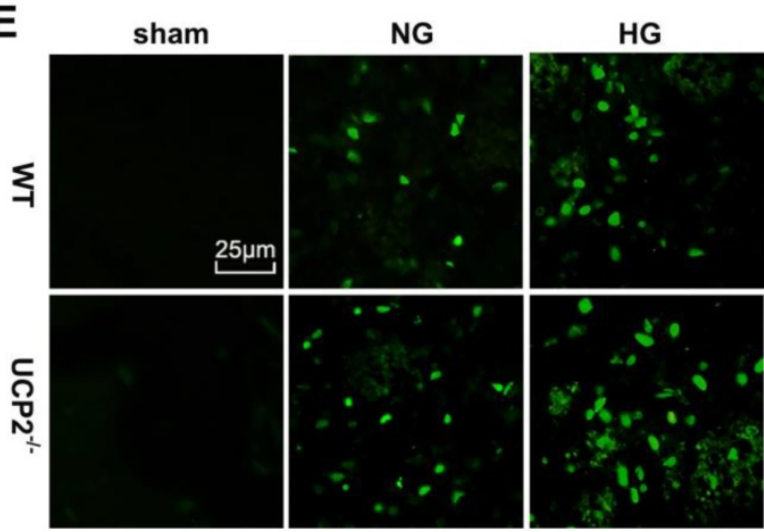

B

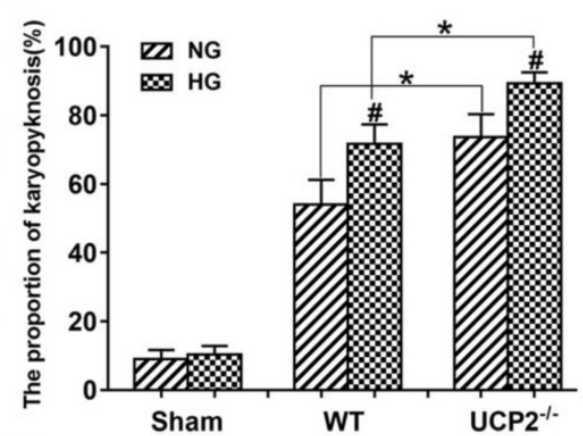

D

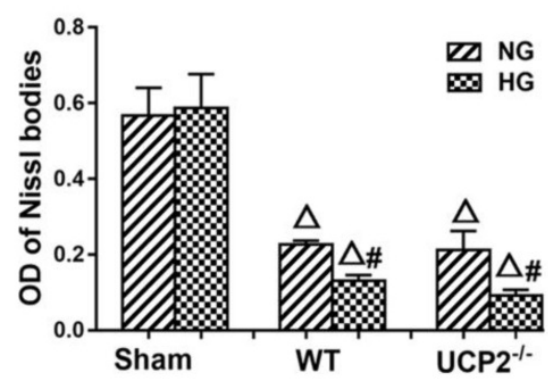

$\mathbf{F}$

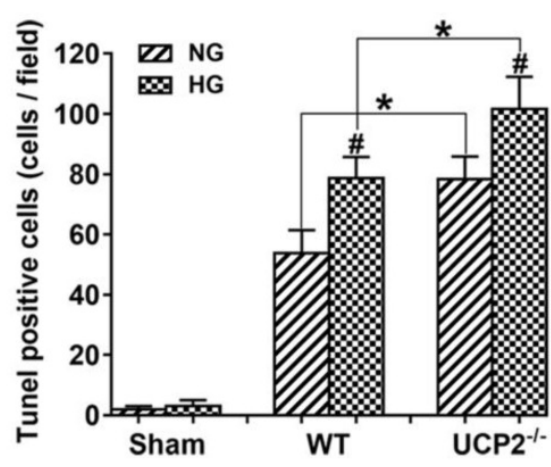

Figure 3. UCP2 deletion aggravated ischemia/reperfusion induced histopathological changes and apoptotic in hyperglycemic mice. (A) HE staining. (B) Quantitative summary of pyknotic cells. (C) Nissl staining. (D) The average optical density of each group of Nissl bodies. (E) Apoptosis was determined via TUNEL test. (F) the number of TUNEL positive cells ( $n=6$ mice, each group). ${ }^{\Delta} p<0.05$ vs. sham group; ${ }^{*} p<0.05$ vs. WT group; $\# p<0.05$ vs. NG group, respectively. 
animals and at $24 \mathrm{~h}$ and $72 \mathrm{~h}$ in hyperglycemic ischemic animals (Fig. 6B and 6D), suggesting that deletion of UCP2 suppressed mitochondrial fusion. Western blot results showed that ischemia in WT suppressed Opa1 at $24 \mathrm{~h}$ and $72 \mathrm{~h}$ of reperfusion (Fig. $6 \mathrm{E}-6 \mathrm{G})$. The deletion of UCP2 in normoglycemic mice led to an early reduction of Opa1 in normoglycemic animals (Fig. 6E and 6F). Hyperglycemia resulted in a more pronounced decline of Opalin WT than in normoglycemic group in all endpoints including non-ischemic control; however, it did not further decrease Opa1 in UCP2\% mice. Opa1 level in hyperglycemic animals was lower than that of the normoglycemic animals as revealed by two-way ANOVA analysis $(p<0.05)$. The protein level of Mfn2 in the mitochondrial fraction decreased after cerebral ischemia and reperfusion in normoglycemic animals (Fig.6E and 6G). Overall, the Opa1 and Mfn2 levels were intended to be lower in hyperglycemic ischemia than in normoglycemic ischemia and deletion of UCP2 affected these fusion markers in normoglycemic ischemic mice.

To better distinguish the effects of UCP2 knockout on the dynamic balance of mitochondrial fission and fusion, we summarized the levels of mitochondrial fission and fusion markers, calculated the ratio of fission and fusion, and expressed the data as the mitochondrial fission/fusion index. As shown in Figure 7, the fission/fusion index began to rise and peaked at $24 \mathrm{~h}$ in UCP2 $/$ - mice, while mitochondrial fission/fusion index began to rise at $24 \mathrm{~h}$ and reached its peak at $72 \mathrm{~h}$ in WT mice under normoglycemic condition. The finding indicates that in normoglycemic animals, deletion of UCP2 induced mitochondrial fission/fusion index to reach the peak early and is significantly higher than the WT group. In the hyperglycemic group, mitochondrial fission/fusion index began to increase at $6 \mathrm{~h}$ and peaked at $24 \mathrm{~h}$. The ratio decreased slightly in both WT and UCP2 mice at 72h. Mitochondrial fission/fusion index in UCP2/- group was significantly higher than that of the WT group at $24 \mathrm{~h}$ of reperfusion. These indicated that deletion of UCP2 increased mitochondrial fission/fusion index, tuning the mitochondrial dynamic towards fission. Thus, mitochondrial fission/fusion index was higher in UCP2 $\%$ - as compared to WT animals (Fig. 7).

Double labeling of the above fission and fusion markers with neuronal marker NeuN (Fig 8A) or astrocyte marker GFAP (Fig 8B) on brain sections 1 day after reperfusion revealed that Drp1, Fis1, Opa1, and Mfn2 were co-localized with NeuN positive neurons, but not with GFAP positive astrocytes, suggesting that mitochondrial fission and fusion occurred majorly in neurons rather than astrocytes.

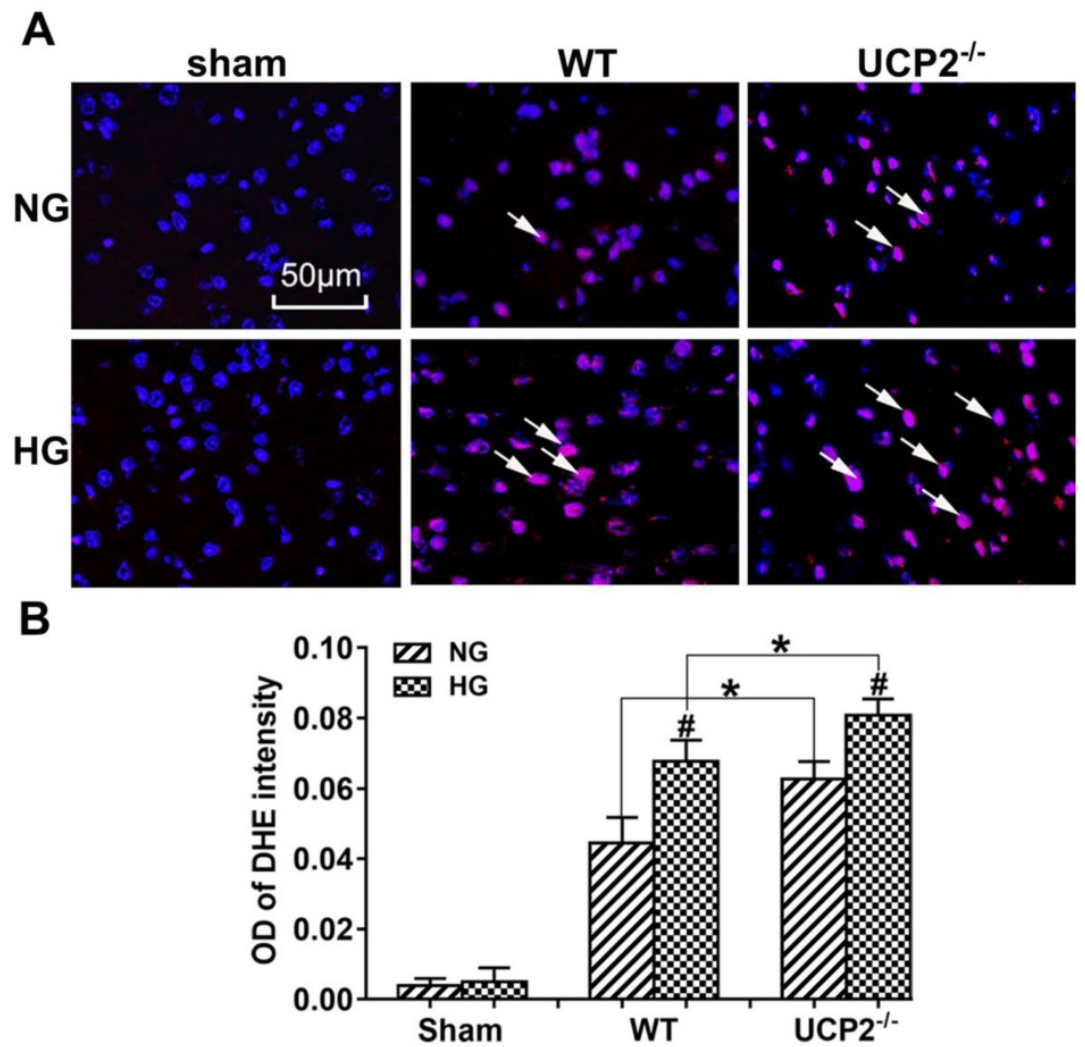

Figure 4. UCP2 deletion enhanced ROS production in hyperglycemic mice. (A) ROS production detected by DHE in WT and UCP2 ${ }^{-/}$mice. Nuclei were labeled with DAPI. Magnification, $400 X$. Scale bar $=50 \mu \mathrm{m}$. (B) summarized DHE fluorescent intensity. $n=4$ mice per group. Data are presented as means \pm SD. ${ }^{*} p<0.001$ vs. WT group in same glucose and $\# p<0.05$ vs. NG group in some type of animal. 


\section{Mitochondrial ultrastructural alterations}

To further demarcate the effect of UCP2 on mitochondria, we performed transmission electron microscope to analyze the mitochondrial ultrastructural changes. Mitochondrial morphology was normal in both WT and UCP2/- non-ischemic control mice. Mild mitochondrial swollen, as reflected by mitochondrial lucency and cristae disarray, was observed in both normoglycemic and hyperglycemic animals after $6 \mathrm{~h}$ of recovery (Fig. 9, red arrows in
$\mathrm{WT}+\mathrm{NG}$ and $\mathrm{WT}+\mathrm{HG}$ images). In contrast, deletion of $\mathrm{UCP} 2$ resulted in markedly mitochondrial swollen, as reflected by increased mitochondrial size, prominent lucency and cristae disarrangement, in both normoand hyperglycemic ischemic animals after $6 \mathrm{~h}$ of reperfusion (Fig. 9, red arrows in $\mathrm{UCP} 2^{-} /+\mathrm{NG}$ and $\mathrm{UCP} 2-/-+\mathrm{HG}$ images). These results indicate that homozygous deletion of UCPs gene aggravates mitochondrial structural damage after ischemia and reperfusion in the brain.
A
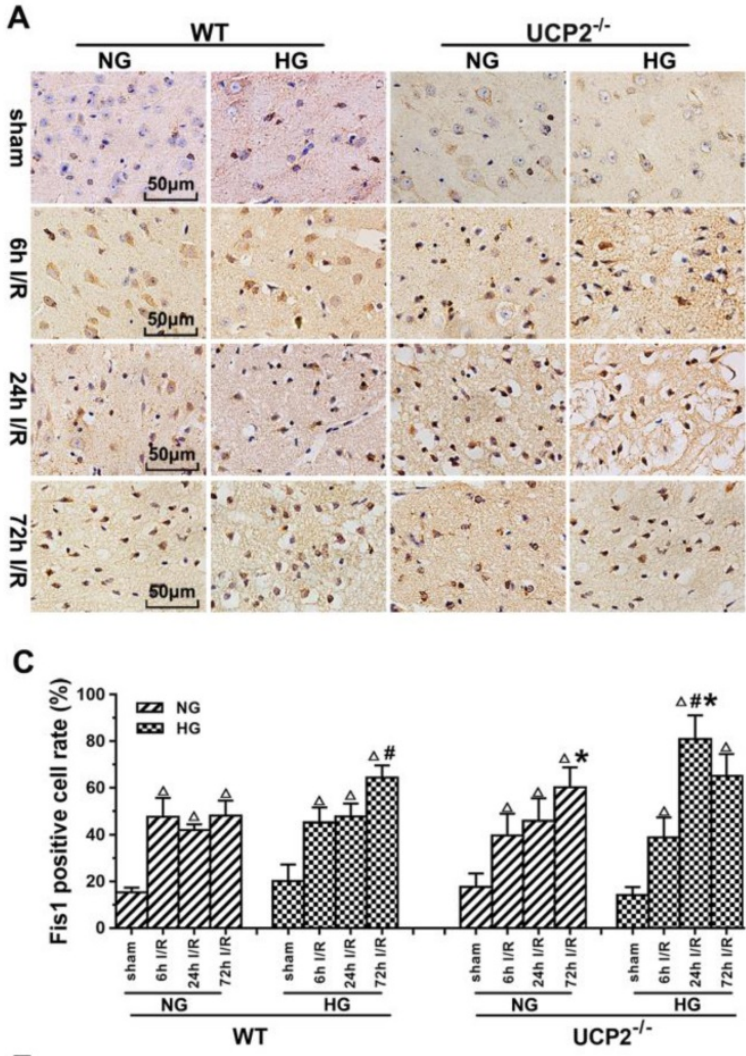

$E$

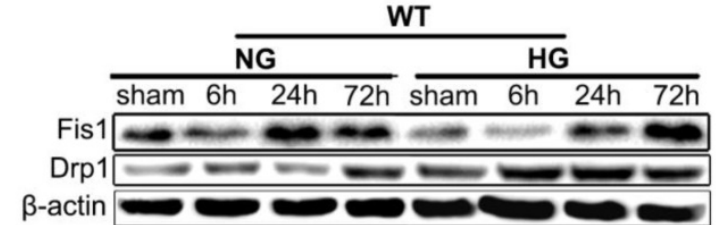

$\mathbf{F}$

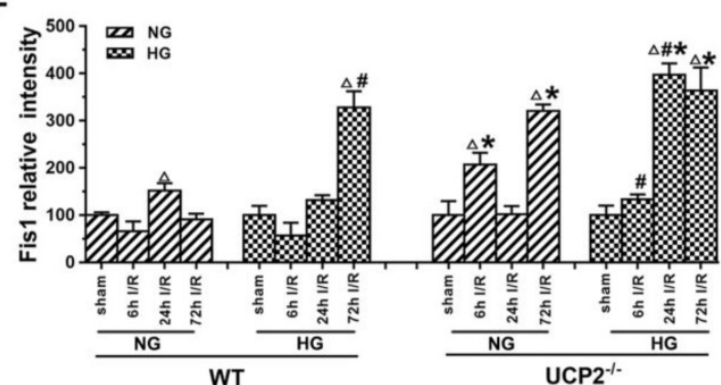

B

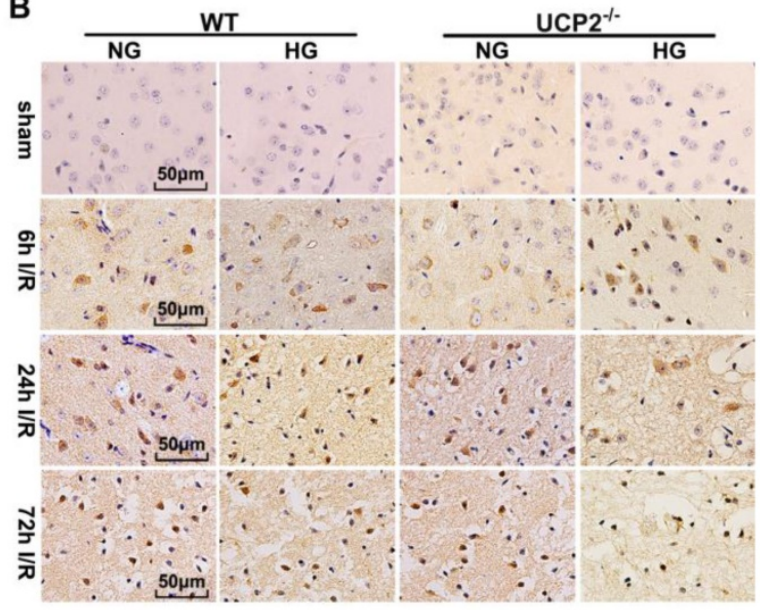

D
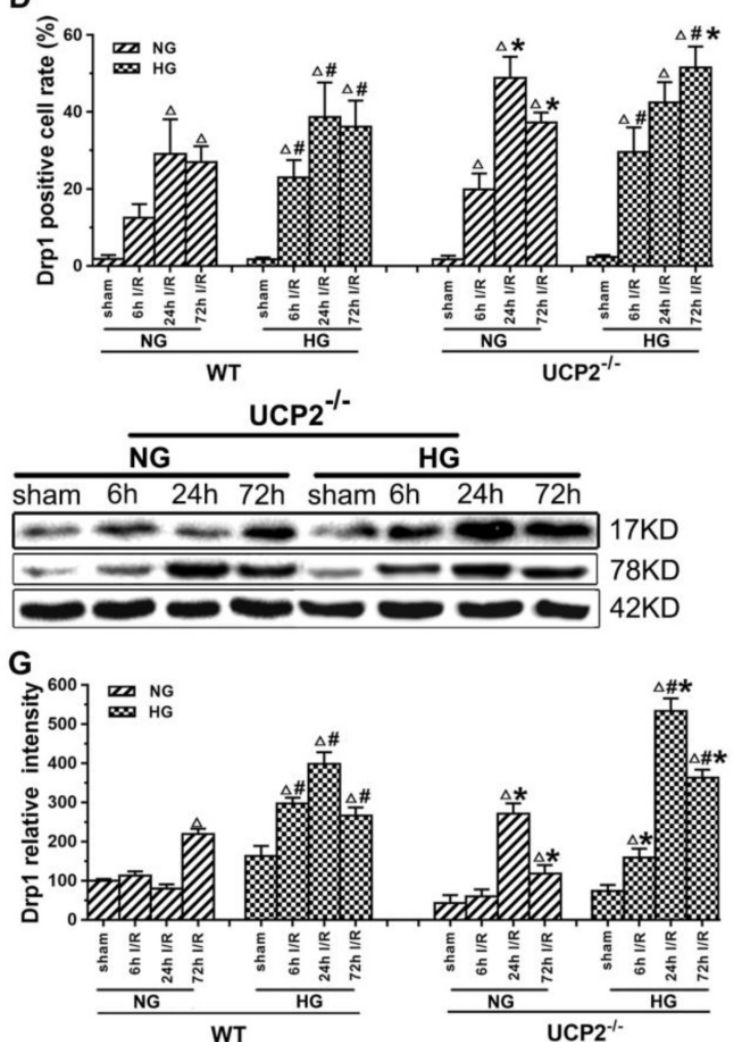

Figure 5. UCP2 deletion increased the levels of mitochondrial fission-related proteins in hyperglycemic mice after ischemia/reperfusion. Detections of mitochondrial fission-related proteins by immunohistochemistry and Western blotting ( $n=6$, per group). (A, B) Representative photomicrographs for Fis 1 and Drp1. Scale bar $=50$ $\mu \mathrm{m}$. (C, D) Quantification of Fis 1 and Drpl immunointensity. E: Representative Western blots for Fis 1 and Drpl. (F, G) Semi-quantification of Fis1 and Drpl protein bands. Data are shown as mean $\pm S D$. For values in $F$ and $G$, the values in $W T+N G$ sham group were converted to 100 and percent changes were presented for other groups relative to the WT+NG sham. ${ }^{\Delta} p<0.05$ vs. respective sham-operated controls, ${ }^{*} p<0.05$ vs. normoglycemic and $* p<0.05$ vs. WT counterpart at an identical reperfusion stage. 
A

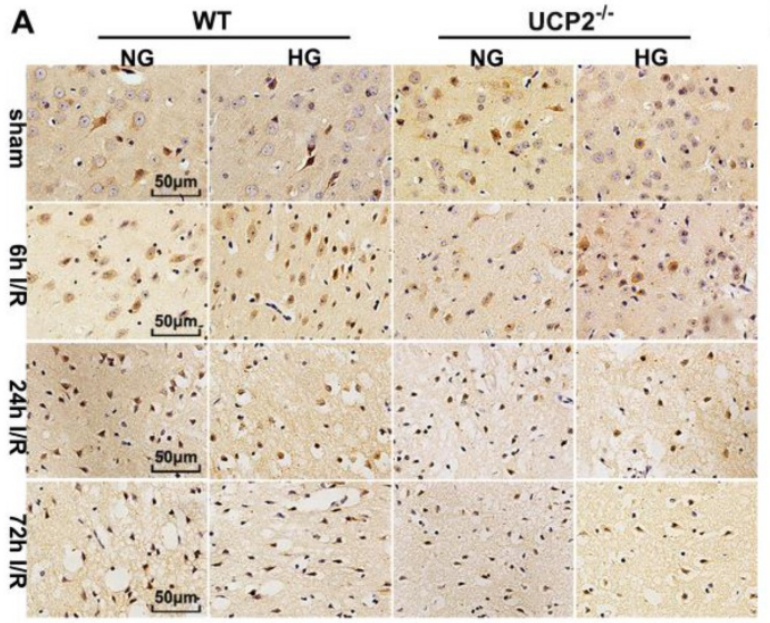

C

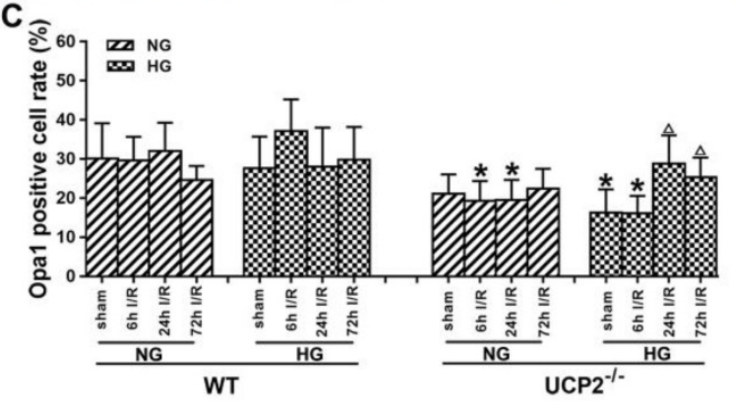

E

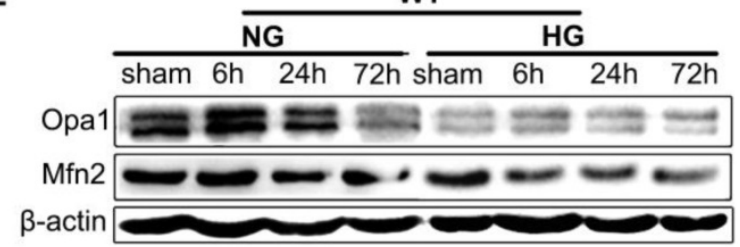

$F$

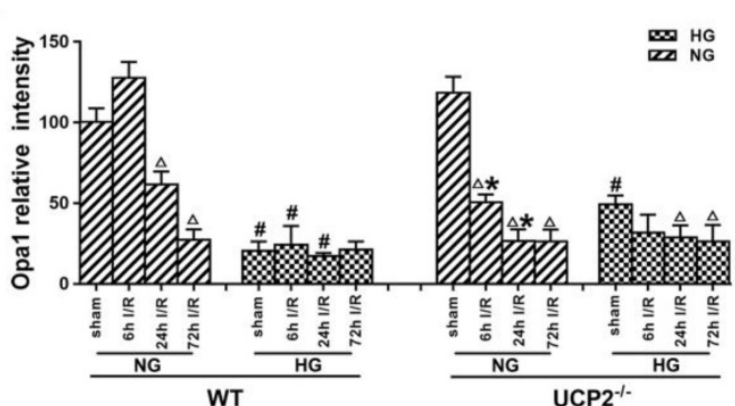

B

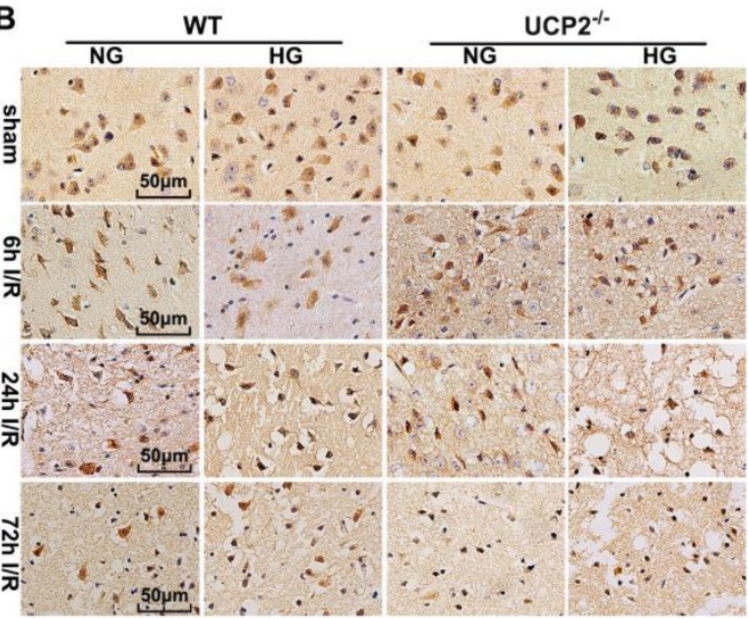

D

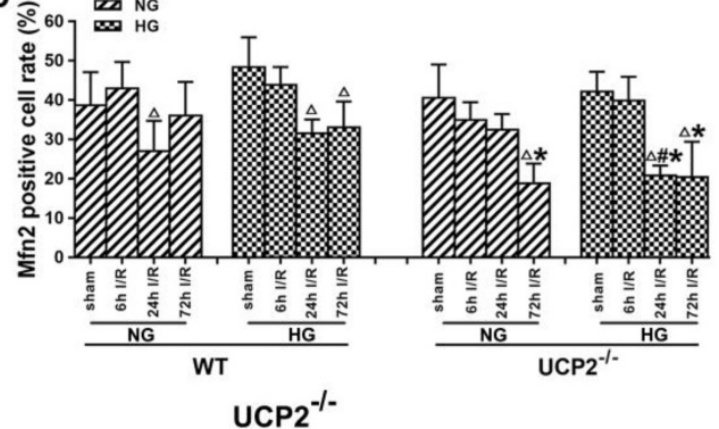

G

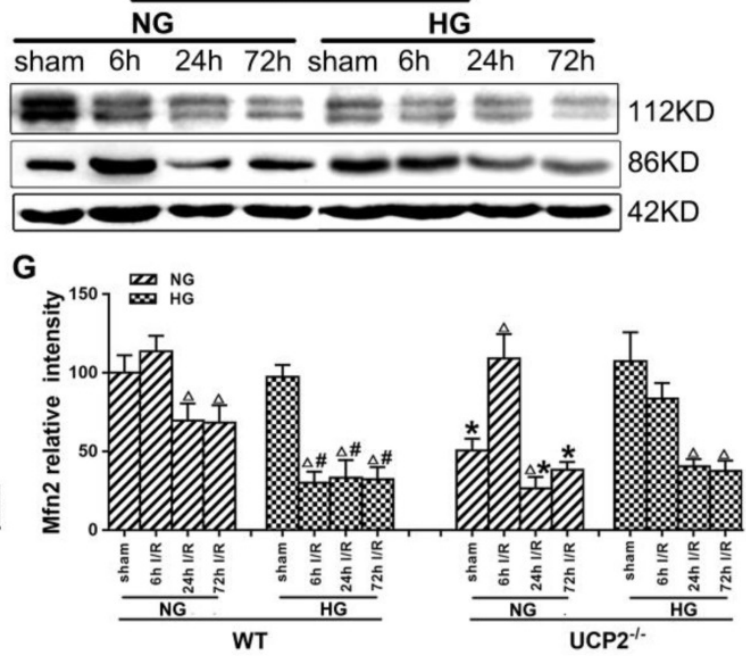

Figure 6. UCP2 deletion increased the levels of mitochondrial fusion-related proteins in hyperglycemic mice after ischemia/reperfusion. Detections of mitochondrial fusion-related proteins by immunohistochemistry and Western blotting. (A, B) Representative photomicrographs for Mfn2 and OPA1. Scale bar=50 $\mu$ m. (C, D) Quantification of Mfn2 and OPA1 immunointensity. E: Representative Western blots for Mfn2 and OPA1. (F, G) Semi-quantification of Mfn2 and OPA1 protein bands. $n=6$ mice per group. Data are shown as mean \pm SD. For values in $F$ and $G$, the values in WT+NG sham group were converted to 100 and percent changes were presented for other groups relative to the $W T+N G$ sham. ${ }^{\Delta} p<0.05$ vs. respective sham-operated controls, ${ }^{\#} p<0.05$ vs. normoglycemic and ${ }^{*} p<0.05$ vs. WT counterpart at an identical reperfusion stage.

\section{Discussion}

Our data demonstrated that the genetic ablation of UCP2 significantly increased infarct volume and brain edema and caused more severe neurological deficit scores after ischemia/reperfusion in both normo- and hyperglycemic animals compared with WT mice subjected to the identical length of ischemia and reperfusion. Further, UCP2 deletion enhanced
ROS production in both normo- and hyperglycemic ischemic mice. Moreover, deletion of UCP2 altered mitochondrial dynamic balance by tilting the balance towards fission, especially in hyperglycemic animals after being subjected to ischemia and reperfusion. Finally, deletion of UCP2 resulted in severe mitochondrial ultrastructural damage that is displayed as increases of mitochondrial size and lucency and disarray of mitochondrial cristae. 


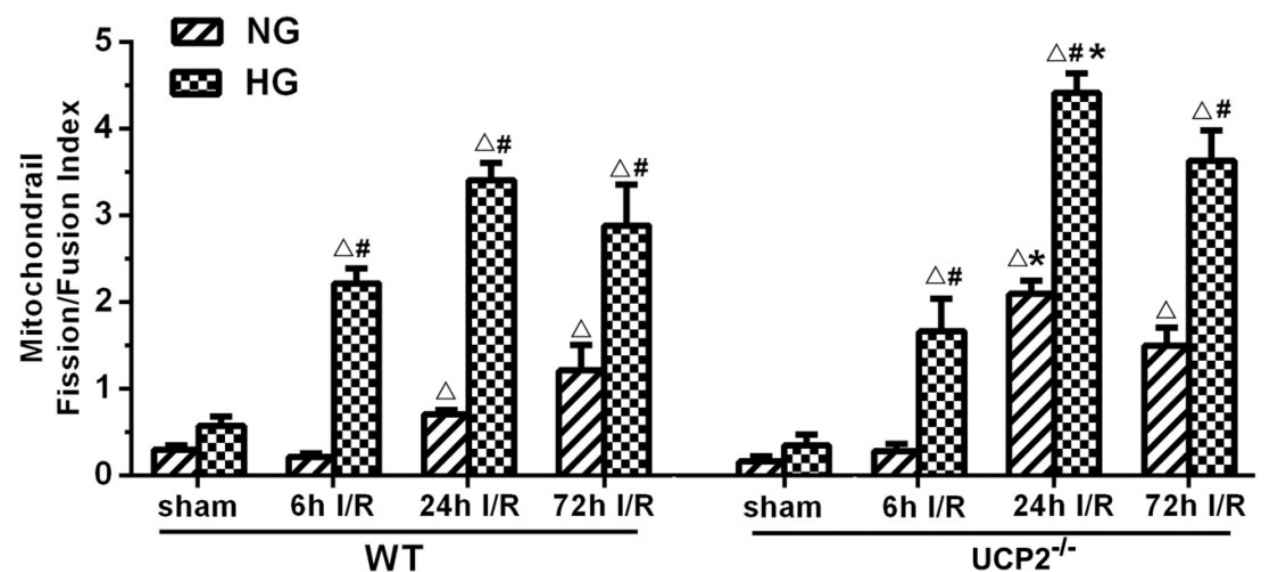

Figure 7. Mitochondrial fission/fusion index following cerebral ischemia and reperfusion under both normo- and hyperglycemic conditions. Index represents the ratio of fission (Drpl and Fisl) and fusion (Opal and Mfn2) proteins. Data are expressed as means \pm SD. $\triangle p<0.05$, vs. respective sham-operated controls, \# $<<0.05$ vs. normoglycemic and $*_{p}<0.05$ vs. WT counterpart at an identical reperfusion stage.
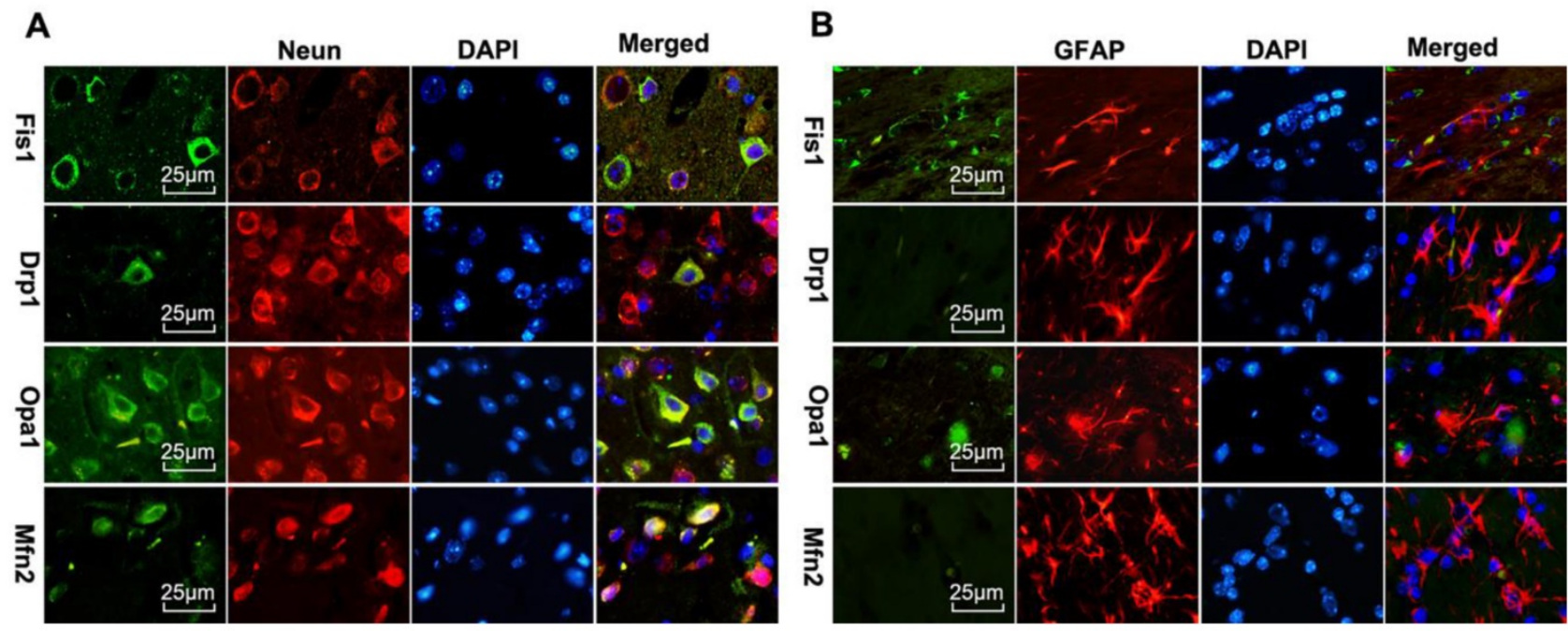

Figure 8. Mitochondrial fusion-related proteins (Opal and Mfn2) and fission-related proteins (Drp1, Fis1) co-localized with neurons in UCP2-- mice following ischemic stroke. Double immunostaining of Drpl, Fis1, Opal and Mfn2 with GFAP (astrocyte marker) and NeuN (neuron marker) were performed in UCP2-- mice brain sections 1 day after reperfusion ( $n=6$, each group). Scale bar $=25 \mu \mathrm{m}$.

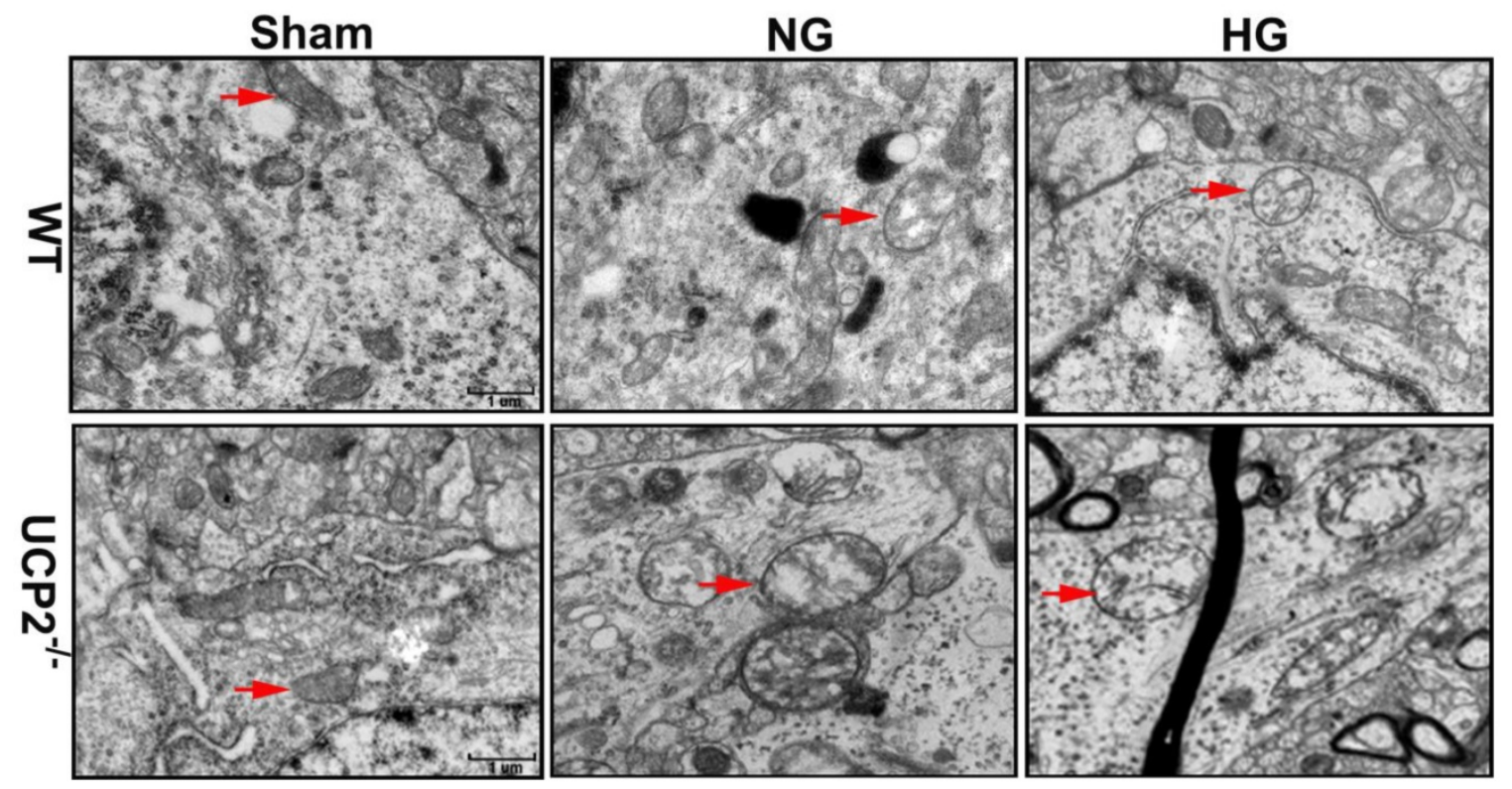

Figure 9. Mitochondrial ultrastructural alterations. Representative transmission electron microscope of neuron form cortical area of the brains in UCP2 ${ }^{-/}$and $\mathrm{WT}$ mice after $6 \mathrm{~h}$ of reperfusion $(\mathrm{n}=4$, each group). Arrow indicates varying mitochondrial size whereas arrowhead shows mitochondrial swelling and disarrayed cristae. Scale bar $=1 \mu \mathrm{m}$. 
UCP2 is an inner mitochondrial membrane protein that dissipates the mitochondrial proton gradient by transporting $\mathrm{H}^{+}$across the inner membrane, generating heat, stabilizing the inner mitochondrial membrane potential and reducing the formation of reactive oxygen species (ROS) [23]. UCP2 is widely expressed in various tissues including the central nervous system and implicated in diverse pathologic conditions such as obesity, diabetes, neurodegenerative diseases, atherosclerosis, and cancer $[24,25,26]$. Emerging evidence suggests that UCP2 may play an important role in cerebral stroke by regulating mitochondrial potential and energy balance, neuroendocrine and autonomic functions, reactive oxygen species (ROS) production and fatty acid anion transport, cell death, and inflammation [27]. Among the published literature, a majority of studies have demonstrated that increasing UCP2 is neuroprotective [28]. Upregulation of UCP2 has been reported to reduce neuronal damage in cerebral stroke, traumatic brain injury, epilepsy and Parkinson's models [6, 7, 29, 30]. Our data demonstrated that the genetic ablation of UCP2 significantly increased infarct volume, brain edema, apoptosis and aggravated neurological deficit scores after ischemia/reperfusion under normoglycemic condition, which is consistent with our previous report and those published in the literature [6,31,32]. Furthermore, our results for the first time demonstrated that deletion of UCP2 further worsened the ischemic brain damage in hyperglycemic animals comparing to wildtype animals subjected to hyperglycemic ischemia. These results suggest that UCP2 plays an important role in cerebral ischemic injury under both normo- and hyperglycemic conditions.

Reactive oxygen species (ROS) are free radicals that can damage DNA, lipids, and proteins. Hyperglycemia has been found to lead to oxidative stress and increased ROS production in neuronal cells $[33,34]$. UCP2 reduces the ROS formation by preventing mitochondrial membrane hyperpolarization that in turn inhibits mitochondrial electron transport chain. Studies have shown that UCP2 confers protective effects on various stressors by decreasing mitochondrial ROS production in the brain and liver [35, 36, 37]. It has also been reported that UCP2 could protect cardiomyocytes from exogenous oxidant stress [38]. In the present study, superoxides were increased after ischemia in normoglycemic animals and further elevated by hyperglycemia. Deletion of UCP2 led to exacerbated levels of ROS after ischemia in both normo- and hyperglycemic animals compared to the counterparts in wildtype animals. These data imply that the increased damage observed in $\mathrm{UCP}^{-} /$mice is partially due to increases in ROS production.

Recent studies have suggested that impaired mitochondrial dynamics and excessive mitochondrial fission are connected to several neurodegenerative disorders such as stroke [39], Parkinson's diseases [40], and Alzheimer's diseases [41]. In the present study, we also found in both immunohistochemistry and Western blotting that UCP2 deletion caused mitochondrial dynamic imbalance after cerebral ischemic injury under both normo- and hyperglycemic conditions. The results demonstrated that ischemia and reperfusion increased the protein levels of fission proteins Fis1 and Drp1, and decreased fusion proteins Opa1and Mfn2, thereby, tilting the mitochondrial dynamic balance towards fission. Preischemic hyperglycemia further augmented the alteration as reflected by the higher levels of Fis1 and Drp1 in hyperglycemic animals at $6 \mathrm{~h}$ of reperfusion than those observed in the normoglycemic counterparts, suggesting that hyperglycemia caused an early onset of mitochondrial fission process by hyperglycemia. This is consistent with our previous finding that hyperglycemic ischemia increases mitochondrial dynamic imbalance towards fission [21]. When comparing to wildtype animals, deletion of UCP2 further increased the levels of Fis1 and Drp1 in both normo- and hyperglycemic ischemic animals. Previous study shows UCP2 in the ventromedial nucleus of the hypothalamus is required for glucose-induced DRP1-mediated mitochondrial fission [42]. However, the impact of UCP2 deletion on mitochondrial fission and fusion balance after cerebral ischemia has not been reported before. A recent study using acute kidney ischemia and reperfusion model demonstrated that UCP knockout mice had pronounced mitochondrial dynamic imbalance towards fission and mitochondrial fragmentation; whereas upregulation of UCP2 ameliorated hypoxia-induced mitochondrial fusion and fission imbalance [43]. The immunoreactivity of Opa1 was not changed after ischemia in both normoand hyperglycemic animals after ischemia and reperfusion up to $72 \mathrm{~h}$. That of the Mfn2 decreased in both normo- and hyperglycemic animals with no significant difference between the two glycemic conditions. In contrast, deletion of UCP2 significantly decreased the immunoreactivity of Opa1 and Mfn2 in both normo- and hyperglycemic ischemic animals, suggesting deletion of UCP2 inhibited mitochondrial fusion. Semi-quantitative measurements of Opa1 and Mfn2 by protein blotting revealed marked decreases of these two fusion proteins after ischemia in normoglycemic animals and the decreases were more pronounced in hyperglycemic ischemic animals than 
in normoglycemic animals, suggesting hyperglycemia inhibited mitochondrial fusion. Deletion of UCP2 further decreased the levels of Opa1 and Mfn2 and the decreases were more obvious in normoglycemic than hyperglycemic animals. The discrepant result of Opa1 between immunohistochemistry and Western blotting may ascribe to the fact that IHC detects the target proteins in specific areas, while ischemic lateral brain was used in Western blotting, which more accurately reflects the changes of fusion protein levels.

Because the general tendency was that ischemia increased fission and decreased fusion proteins, that hyperglycemia further aggravated these alterations, and that UCP2 deletion made the changes even more obvious than in wildtype counterpart animals, we decided to calculate mitochondrial fission/fusion index, which is the ratio of fission proteins divided by fusion proteins. The results clearly demonstrated that ischemia in normoglycemic animals induced mitochondrial fission and hyperglycemia induced a more dramatic increase. These results are further supported by previous publications showing that hyperglycemia causes mitochondrial fission [44, 45]. Deletion of UCP2 further increased the fission/fusion index in both normo-and hyperglycemic animals. These data suggest that hyperglycemic ischemia enhances mitochondrial fission after cerebral ischemia and reperfusion and UCP2 deletion further increases mitochondrial dynamic imbalance under both normoand hyperglycemic conditions. The slanting of the mitochondrial dynamics to fission may reduce the mitochondrial network and enhance mitochondrial rupture and induce neuronal cell death after cerebral ischemia [46]. UCP2 silencing has been shown to cause mitochondrial dysfunction in astrocytes under septic conditions [47]. However, it is not known whether UCP2 deletion affects the mitochondrial dynamics in astrocytes. Based on our double immunostaining results, the fission and fusion protein markers were co-localized with neuronal marker NeuN, but not with astrocytes. This indicated that UCP2 deficiency mainly affects mitochondrial division and fusion balance in neurons.

Mitochondrial dynamic imbalance could result in mitochondrial morphological alterations [48]. Observation of the mitochondrial ultrastructure using an electron microscope found mild to moderately swollen mitochondria with increased lucency and cristae disarrangement in both normo- and hyperglycemic animals as early as $6 \mathrm{~h}$ of reperfusion after $1 \mathrm{~h}$ of ischemia. In UCP2 $\%$ mice, mitochondrial sizes increased significantly due to swelling. Pronounced lucency and disarray and disappearance of mitochondrial cristae were observed, suggesting that UCP2 deletion increases mitochondrial morphological damage caused by cerebral ischemia in both normo- and hyperglycemic animals. We have previously shown that hyperglycemia aggravates ischemic brain damage [49, 50]. This effect is associated with increases of mitochondrial fission and mitochondrial morphological alterations [21, 33, 51]. In this study, we observed mild mitochondrial damage including lucency and disarray of cristae in both normo- and hyperglycemic animals at $6 \mathrm{~h}$ of reperfusion. UCP2 knockout further aggravated the mitochondrial damage in both normo- and hyperglycemic mice after ischemia and reperfusion. Therefore, mitochondrial size was further enlarged and mitochondrial lucency and vacuolization were more prominent than those being observed in wildtype mice. The impact of UCP2 on mitochondrial morphology of the brain tissue after cerebral ischemia and reperfusion has not been reported. Several studies have demonstrated that silencing UCP2 by small interference RNA resulted in more severe mitochondrial swelling, vacuolization and loss of matrix content in cardiomyocytic $\mathrm{H} 9 \mathrm{C} 2$ cells and astrocytes compared with control cells challenged with sepsis [47, 52, 53]. These results support our finding that UCP2 plays an important role in maintaining mitochondrial dynamic and morphological integrity in the brain.

Collectively, deletion of UCP2 has an adverse impact on cerebral ischemia-reperfusion injury under both normo- and hyperglycemic conditions. Deletion of UCP2 increases ROS production, mitochondrial fission and morphological abnormalities. These findings may pave the way to new treatment modalities, which are needed for the treatment of brain I/R injury.

\section{Abbreviations}

UCPs: Uncoupling proteins; UCP2: Uncoupling protein 2; UCP2--: UCP2 deletion; I/R: ischemia/reperfusion; MCAO: middle cerebral artery occlusion; WT: wildtype; NG: normoglycemic; HG: hyperglycemic; TUNEL: Terminal deoxynucleotidyl transferase mediated dUTP nick-end labeling; ROS: reactive oxygen species; Fis1: fission 1; Drp1: dynamin-related protein 1; Opa1: optic atrophy 1; Mfn2: mitofusin 2; STZ: Streptozotocin; TTC: 2,3,5-Triphenyl Tetrazolium Chloride; ICA: internal carotid artery; ECA: external carotid artery; CCA: common carotid artery; DHE: Dihydroethidium.

\section{Acknowledgements}

The authors greatly appreciate Ms. Roslyn Lewis for language checking and proof reading. This study was supported by the Natural Science Foundation of Ningxia Hui Autonomous Region (2019AAC03212) to 
Maotao He, the Ningxia Medical University Research Fund (XM2016028) to Maotao He and the National Science Foundation of China (No. 81560208) to Li Jing. The funders had no role in study design, data collection and analysis, decision to publish, or preparation of the manuscript.

\section{Author Contributions}

P. Andy Li and Li Jing conceived and designed the experiments. Maotao He, Yanmei $\mathrm{Ma}$ and Rui Wang performed the experiments. Maotao $\mathrm{He}$ and Jianzhong Zhang analyzed the data. Maotao He prepared the writing-original draft. P. Andy Li and Li Jing reviewed and edited the manuscript.

\section{Competing Interests}

The authors have declared that no competing interest exists.

\section{References}

1. Toda C, Diano S. Mitochondrial UCP2 in the central regulation of metabolism. Best Pract Res Clin Endocrinol Metab. 2014; 28(5): 757-764.

2. Lee S, Moon H, Kim G, Cho JH, Lee DH, Ye MB, Park D. Anion transport or nucleotide binding by UCP2 is indispensable for UCP2-mediated efferocytosis. Mol Cells. 2015; 38(7): 657.

3. Islam R, Yang L, Sah M, Kannan K, Anamani D, Vijayan C, Fridell YW. A neuroprotective role of the human uncoupling protein 2 (hUCP2) in a Drosophila Parkinson's disease model. Neurobiol Dis. 2012; 46(1): 137-146.

4. Dutra MRH, Feliciano RDS, Jacinto KR, Gouveia TLF, Brigidio E, Serra AJ, Silva J. Protective role of UCP2 in oxidative stress and apoptosis during the silent phase of an experimental model of epilepsy induced by pilocarpine. Oxid Med Cell Longev. 2018; 2018.

5. Bhattacharya R, Singh P, John JJ, Gujar NL. Oxidative damage mediated iNOS and UCP-2 upregulation in rat brain after sub-acute cyanide exposure: dose and time-dependent effects. Drug Chem Toxicol. 2019; 42(6): 577-584.

6. Haines BA, Mehta SL, Pratt SM, Warden CH, Li PA. Deletion of mitochondrial uncoupling protein-2 increases ischemic brain damage after transient focal ischemia by altering gene expression patterns and enhancing inflammatory cytokines. J Cereb Blood Flow Metab. 2010; 30(11): 1825-1833.

7. Haines B, Li PA. Overexpression of mitochondrial uncoupling protein 2 inhibits inflammatory cytokines and activates cell survival factors after cerebral ischemia. PLoS One. 2012; 7(2): e31739.

8. Chen R, Ovbiagele B, Feng W. Diabetes and stroke: epidemiology, pathophysiology, pharmaceuticals and outcomes. Am J Med Sci. 2016; 351(4): 380-386

9. Tun NN, Arunagirinathan G, Munshi SK, Pappachan JM. Diabetes mellitus and stroke: A clinical update. World J Diabetes. 2017; 15; 8(6):235-248.

10. Shukla V, Shakya AK, Perez-Pinzon MA, Dave K R. Cerebral ischemic damage in diabetes: an inflammatory perspective. J Neuroinflammation. 2017; 14(1): $1-22$

11. He Q, Csiszar K, Li PA. Transient forebrain ischemia induced phosphorylation of cAMP-responsive element-binding protein is suppressed by hyperglycemia. Neurobiol Dis. 2003; 12(1): 25-34.

12. Muranyi M, Fujioka M, He Q, Han A, Yong G, Csiszar K, Li PA. Diabetes activates cell death pathway after transient focal cerebral ischemia. Diabetes. 2003; 52(2): 481-486

13. Liu P, Yang X, Hei C, Meli Y, Niu J, Sun T, Li PA. Rapamycin reduced ischemic brain damage in diabetic animals is associated with suppressions of mTOR and ERK1/2 signaling. Int J Biol Sci. 2016; 12(8): 1032.

14. Hei C, Liu P, Yang X, Niu J, Li PA. Inhibition of mTOR signaling confers protection against cerebral ischemic injury in acute hyperglycemic rats. Int J Biol Sci. 2017; 13(7): 878.

15. Yang X, Hei C, Liu P, Li PA. Involvement of obesity-associated upregulation of chemerin/chemokine-like receptor 1 in oxidative stress and apoptosis in ovaries and granulosa cells. Biochem Biophys Res Commun. 2019; 510(3): $449-455$

16. Chen H, Chan DC. Mitochondrial dynamics-fusion, fission, movement, and mitophagy-in neurodegenerative diseases. Hum Mol Genet. 2009; 18(R2): R169-R176.

17. Peng C, Rao W, Zhang L, Wang K, Hui H, Wang L, Su N, Luo P, Hao YL, Tu Y, Zhang S, Fei Z. Mitofusin 2 ameliorates hypoxia-induced apoptosis via mitochondrial function and signaling pathways. Int J Biochem Cell Biol. 2015; 69: 29-40.
18. Barsoum, MJ, Yuan $\mathrm{H}$, Gerencser A A, Liot G, Kushnareva Y, Gräber S, White AD. Nitric oxide-induced mitochondrial fission is regulated by dynaminrelated GTPases in neurons. EMBO J. 2006; 25(16): 3900-3911.

19. Liu W, Tian F, Kurata T, Morimoto N, Abe K. Dynamic changes of mitochondrial fusion and fission proteins after transient cerebral ischemia in mice. J Neurosci Res. 2012; 90(6): 1183-1189.

20. Martorell-Riera A, Segarra-Mondejar M, Muñoz JP, Ginet V, Olloquequi J, Pérez-Clausell J, Soriano FX. Mfn2 downregulation in excitotoxicity causes mitochondrial dysfunction and delayed neuronal death. EMBO J. 2014; 33(20): 2388-2407.

21. Kumari S, Anderson L, Farmer S, Mehta SL, Li PA. Hyperglycemia alters mitochondrial fission and fusion proteins in mice subjected to cerebral ischemia and reperfusion. Transl Stroke Res. 2012; 3(2): 296-304.

22. Li PA, Shamloo M, Katsura KI, Smith ML, Siesjö BK. Critical values for plasma glucose in aggravating ischaemic brain damage: correlation to extracellular pH. Neurobiol Dis. 1995; 2(2): 97-108.

23. Ji F, Shen T, Zou W, Jiao J. UCP2 Regulates Embryonic Neurogenesis via ROSMediated Yap Alternation in the Developing Neocortex. Stem Cells. 2017; 35(6): 1479-1492.

24. Donadelli M, Dando I, Dalla Pozza E, Palmieri M. Mitochondrial uncoupling protein 2 and pancreatic cancer: a new potential target therapy. World J Gastroenterol. 2015; 21;21(11):3232-8.

25. Li J, Jiang R, Cong X, Zhao Y. UCP 2 gene polymorphisms in obesity and diabetes, and the role of UCP 2 in cancer. FEBS letters. 2019; 593(18): 2525-2534.

26. Sreedhar A, Zhao Y. Uncoupling protein 2 and metabolic diseases. Mitochondrion. 2017; 34: 135-140.

27. Duffy $\mathrm{CM}, \mathrm{Xu} \mathrm{H}$, Nixon JP, Bernlohr DA, Butterick TA. Identification of a fatty acid binding protein4-UCP2 axis regulating microglial mediated neuroinflammation. Mol Cell Neurosci. 2017; 80: 52-57.

28. Mehta SL, Li PA. Neuroprotective role of mitochondrial uncoupling protein 2 in cerebral stroke. J Cereb Blood Flow Metab. 2009; 29(6): 1069-1078.

29. Normoyle KP, Kim M, Farahvar A, Llano D, Jackson K, Wang H. The emerging neuroprotective role of mitochondrial uncoupling protein-2 in traumatic brain injury. Transl Neurosci. 2015; 6(1).

30. Kishimoto Y, Johnson J, Fang W, Halpern J, Marosi K, Liu D, Geisler JG, Mattson MP. A mitochondrial uncoupler prodrug protects dopaminergic neurons and improves functional outcome in a mouse model of Parkinson's disease. Neurobiol Aging. 2020; 85: 123-130.

31. Mattiasson G, Shamloo M, Gido G, Mathi K, Tomasevic G, Yi S, Nikolich K. Uncoupling protein-2 prevents neuronal death and diminishes brain dysfunction after stroke and brain trauma. Nat Med. 2003; 9(8): 1062-1068.

32. Deierborg T, Wieloch T, Diano S, Warden $\mathrm{CH}$, Horvath TL, Mattiasson G. Overexpression of UCP2 protects thalamic neurons following global ischemia in the mouse. J Cereb Blood Flow Metab. 2008; 28(6): 1186-1195.

33. Muranyi M, Ding C, He Q, Lin Y, Li PA. Streptozotocin-induced diabetes causes astrocyte death after ischemia and reperfusion injury. Diabetes. 2006; 55(2): 349-355

34. Kaeidi A, Hajializadeh Z, Jahandari F, Fatemi I. Leptin attenuates oxidative stress and neuronal apoptosis in hyperglycemic condition. Fundam Clin Pharmacol. 2019; 33(1): 75-83.

35. Streck EL, Gonçalves CL, Furlanetto CB, Scaini G, Dal-Pizzol F, Quevedo J. Mitochondria and the central nervous system: searching for a pathophysiological basis of psychiatric disorders. Braz J Psychiatry. 2014; 36(2): 156-167.

36. Hass DT, Barnstable CJ. Uncoupling protein 2 in the glial response to stress: implications for neuroprotection. Neural Regen Res. 2016; 11(8): 1197.

37. Zhong H, Liu M, Ji Y, Ma M, Chen K, Liang T, Liu C. Genipin reverses HFD-induced liver damage and inhibits UCP2-mediated pyroptosis in mice. Cell Physiol Biochem. 2018; 49(5): 1885-1897.

38. Wu H, Ye M, Liu D, Yang J, Ding JW, Zhang J, Yang J. UCP2 protect the heart from myocardial ischemia/reperfusion injury via induction of mitochondrial autophagy. J Cell Biochem. 2019; 120(9): 15455-15466.

39. Yang JL, Mukda S, Chen SD. Diverse roles of mitochondria in ischemic stroke. Redox Biol. 2018; 16: 263-275.

40. Von Stockum S, Nardin A, Schrepfer E, Ziviani E. Mitochondrial dynamics and mitophagy in Parkinson's disease: A fly point of view. Neurobiol Dis. 2016; 90: 58-67.

41. Hroudová J, Singh N, Fišar Z. Mitochondrial dysfunctions in neurodegenerative diseases: relevance to Alzheimer's disease. Biomed Res Int. 2014; 2014.

42. Toda C, Kim JD, Impellizzeri D, Cuzzocrea S, Liu ZW, Diano S. UCP2 regulates mitochondrial fission and ventromedial nucleus control of glucose responsiveness. Cell. 2016; 164(5): 872-883.

43. Qin N, Cai T, Ke Q, Yuan Q, Luo J, Mao X, Zhou Y. UCP2-dependent improvement of mitochondrial dynamics protects against acute kidney injury. J pathology. 2019; 247(3): 392-405.

44. Kumari S, Mehta SL, Li PA. Glutamate induces mitochondrial dynamic imbalance and autophagy activation: preventive effects of selenium. PloS one. 2012; 7(6): e39382.

45. Klacanova K, Kovalska M, Chomova M, Pilchova I, Tatarkova Z, Kaplan P, Racay P. Global brain ischemia in rats is associated with mitochondrial release and downregulation of Mfn2 in the cerebral cortex, but not the hippocampus. Int J Mol Med. 2019; 43(6): 2420-2428.

46. Friedman J R, Nunnari J. Mitochondrial form and function. Nature. 2014; 505(7483): 335-343. 
47. Peng W, Huang J, Zheng Y, Ding Y, Li S, Zhang J, Lyu J, Zeng Q. UCP2 silencing aggravates mitochondrial dysfunction in astrocytes under septic conditions. Mol Med Rep. 2019; 20: 4459-4466.

48. Saita S, Ishihara T, Maeda M, Iemura SI, Natsume T, Mihara K, Ishihara N. Distinct types of protease systems are involved in homeostasis regulation of mitochondrial morphology via balanced fusion and fission. Genes Cells. 2016; 21(5): 408-424.

49. Liu, WJ, Jiang HF, Rehman FU, Zhang JW, Chang Y, Jing L, Zhang JZ. Lycium barbarum polysaccharides decrease hyperglycemia-aggravated ischemic brain injury through maintaining mitochondrial fission and fusion balance. J Biol Med Sci. 2017; 13(7): 901.

50. Lu CJ, Guo YZ, Zhang Y, Yang L, Chang Y, Zhang JW, Zhang JZ. Coenzyme Q10 ameliorates cerebral ischemia reperfusion injury in hyperglycemic rats. Pathol Res Pract. 2017; 213(9): 1191-1199.

51. Ding C, He QP, Li PA. Activation of cell death pathway after a brief period of global ischemia in diabetic and non-diabetic animals. Exp Neurol. 2004; 188(2): $421-429$

52. Zheng G, Lyu J, Liu S, Huang J, Liu C, Xiang D, Zeng Q. Silencing of uncoupling protein 2 by small interfering RNA aggravates mitochondrial dysfunction in cardiomyocytes under septic conditions. Int J Mol Med. 2015; 35(6): 1525-1536.

53. Huang J, Peng W, Zheng Y, Hao H, Li S, Yao Y, Zeng Q. Upregulation of UCP2 Expression Protects against LPS-Induced Oxidative Stress and Apoptosis in Cardiomyocytes. Oxid Med Cell Longev. 2019; 2019. 